\title{
First Organocatalyzed Asymmetric Michael Addition of Aldehydes to Vinyl Sulfones
}

\author{
Sarah Mossé and Alexandre Alexakis* \\ Department of Organic Chemistry, University of Geneva, 30, quai Ernest Ansermet, CH-1211 \\ Geneva 4, Switzerland
}

Supporting Information

General Remarks. All reactions were carried out under a nitrogen atmosphere with oven dried glassware. Concerning ${ }^{1} \mathrm{H}(400 \mathrm{MHz})$ and ${ }^{13} \mathrm{C}(100 \mathrm{MHz}) \mathrm{NMR}$ spectra, chemical shift $(\delta)$ are given in ppm relative to tetramethylsilane (0 ppm). Multiplicity is indicated as follows : s (singlet), d (doublet), t (triplet), q (quartet), m (multiplet), dd (doublet of doublet), br s (broad singlet). Coupling constants are reported in Hz. GC/MS were recorded with Electronic Impact EI (70 eV) as source; m/z (\%). Mass spectra (MS) were obtained by EI (70 eV) and High resolution mass spectra HRMS by Electrospray Ionisation (ESI). Optical rotations were measured at $25^{\circ} \mathrm{C}$ in a $10 \mathrm{~cm}$ cell in the stated solvent ; $[\alpha]_{D}$ values are given in $10^{-1}$ deg.cm ${ }^{2}$ $\mathrm{g}^{-1}$ (concentration c given as $\mathrm{g} / 100 \mathrm{~mL}$ ). Enantiomeric excesses were determined by chiral Super Fluid Chromatography (SFC), with appropriated program using a gradient of methanol or by chiral Gas Chromatography (GC), with appropriated program using a gradient of temperature. Temperature programs are described as follows: initial temperature $\left({ }^{\circ} \mathrm{C}\right)$ - initial time $(\min )$ - temperature gradient $\left({ }^{\circ} \mathrm{C} / \mathrm{min}\right)$ - final temperature $\left({ }^{\circ} \mathrm{C}\right)$; retention times $\left(\mathrm{R}_{\mathrm{T}}\right)$ are given in min. Flash chromatography was performed using florisil ${ }^{\circledR}$ 100-200 mesh or silicagel 32-63 $\mu \mathrm{m}, 60$ Å.

Evolution of reaction was followed by TLC. Chloroform, extra dry, with molecular sieves was purchased from Acros and dried by filtration over basic alumina. Pyrrolidine using in organocatalysis was distilled at atmosphere pressure prior to use. phenylvinyl sulfone $\mathbf{1}$ was purchased from Fluka and used as received. 1,1-bis(benzenesulfonyl)ethylene 2 was purchased from Fluka or synthesized by known experimental procedures ${ }^{1}$ starting from commercially available thiophenol and dichloromethane. Diamine iPBP 4c was prepared according to the literature procedure. ${ }^{2}$

Aldehydes 3a-b,d-h and L-proline were purchased from Fluka and used as received. (S)-(+)(1-pyrrolidinylmethyl)pyrrolidine $\mathbf{4 g}$ diamine was purchased from Aldrich and used as received. 2-cyclohexylacetaldehyde $3 \mathbf{c}$ was prepared according to the literature procedure. ${ }^{3}$

THF and dichloromethane were dried by filtration over activated alumina. Acetonitrile was distilled over $\mathrm{CaH}_{2}$ prior to use. Methanol was used without previous distillation.

\footnotetext{
${ }^{1}$ Preparation of bis(phenylthio)methane, see Screttas, C. G.; Micha-Screttas, M. J. Org. Chem 1979, 44, 713. Preparation of bis(phenylsulfonyl)methane, see Nantz, M. H.; Radisson, X.; Fuchs, P. L. Synth. Commun. 1987, 17, 55. Preparation of 1,1-bis(benzenesulfonyl)ethylene 2 : for the $1^{\text {st }}$ step Setter, see H.; Steinbeck, K. Liebigs Ann. Chem. 1974, 1315. And for the $2^{\text {nd }}$ step, see Carpino, L. A. J. Org. Chem., 1973, 38, 2600.

2 (a) Alexakis, A.; Andrey, O. Org. Lett. 2002, 4, 3611. (b) Andrey, O.; Alexakis, A.; Tomassini, A.; Bernardinelli, G. Adv. Synth. Catal. 2004, 346, 1147.

${ }^{3}$ Rumbolt, G.; Righi, G. J. Org. Chem., 1996, 61, 3557.
} 


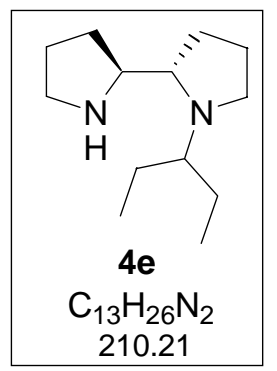

To a solution of 2S,2'S-bipyrrolidine ${ }^{4}$ (435 mg, 3 mmol, 1 eq.) in pentan-3one $(5 \mathrm{~mL})$ was added para-toluenesulfonic acid (1.14 g, $6 \mathrm{mmol}, 2$ eq.). After current procedure for hydrogenation, platinium oxide was introduced (34 $\mathrm{mg}, 0.15 \mathrm{mmol}, 0.05$ eq.), then the reaction was conducted under hydrogene atmosphere $(1 \mathrm{~atm})$. The mixture was filtered on celite after 2 days. The filtrate was diluted with $\mathrm{Et}_{2} \mathrm{O}(10 \mathrm{~mL})$ and hydrolyzed with $\mathrm{NaOH}_{\text {aq. }}$ 30\% (5 mL) and water $(3 \mathrm{~mL})$. The solution was decanted, the aqueous phase was extracted three times with $\mathrm{Et}_{2} \mathrm{O}$. The combined organic phases were dried over $\mathrm{MgSO}_{4}$, filtered, concentrated under reduced pressure and purified by bulp to bulp distillation ( $\left.\mathrm{P}=0.3 \mathrm{mbar}, \theta=140^{\circ} \mathrm{C}\right)$ to give the diamine $4 \mathbf{e}(121 \mathrm{mg}, 20 \%)$. $[\alpha]_{\mathrm{D}}=-52.35\left(\mathrm{CHCl}_{3}, \mathrm{c}=0.96 \mathrm{~g} / 100 \mathrm{~mL}\right)$.

${ }^{1} \mathrm{H}$ NMR (400 MHz, $\left.\mathrm{C}_{6} \mathrm{D}_{6}\right): \delta=2.96-2.82(\mathrm{~m}, 3 \mathrm{H}) ; 2.81-2.72(\mathrm{~m}, 3 \mathrm{H}) ; 2.67-2.43(\mathrm{~m}, 1 \mathrm{H})$; 1.70-1.66 (m, 8H); 1.45-1.31 (m, 5H); 1.08 (t, 3H; J = 10.9 Hz); 0.85 (t, 3H, J = 7.6 Hz).

${ }^{13} \mathrm{C}$ NMR $\left(100 \mathrm{MHz}, \mathrm{C}_{6} \mathrm{D}_{6}\right): \delta=63.48(\mathrm{CH}) ; 63.11(\mathrm{CH}) ; 61.88(\mathrm{CH}) ; 45.48\left(\mathrm{CH}_{2}\right) ; 44.21$ $\left(\mathrm{CH}_{2}\right) ; 27.96\left(\mathrm{CH}_{2}\right) ; 26.93\left(\mathrm{CH}_{2}\right) ; 24.69\left(\mathrm{CH}_{2}\right) ; 24.12\left(\mathrm{CH}_{2}\right) ; 23.67\left(\mathrm{CH}_{2}\right) ; 20.14\left(\mathrm{CH}_{2}\right) ; 11.03$ $\left(\mathrm{CH}_{3}\right) ; 10.71\left(\mathrm{CH}_{3}\right)$.

IR $\left(\mathrm{CHCl}_{3}\right)$ : 2956 (s); 2924 (s); 2870 (s); 2814 (m); 1460 (m); 1446 (m); 1376 (w); 1261 (w); 1198 (w); 1159 (w); 1092 (m); 1067 (m); 921 (m); 851 (m).

MS (EI mode) m/z (\%) : 211 (1); 141 (38); 140 (100); 112 (16); 70 (84).

HRMS (ESI) calcd for $\mathrm{C}_{13} \mathrm{H}_{26} \mathrm{~N}_{2}[\mathrm{M}+\mathrm{H}]^{+}$211.2167880, found 211.21679.

GC/MS (80-1-20-270-3) : $\mathrm{R}_{\mathrm{T}}=7.91 \mathrm{~min}$.

Addition of unmodified aldehydes to 1,1-bis(benzenesulfonyl)ethylene 2 catalysed by amines. General Procedure 1 : To a solution of pyrrolidine 4a $(0.16 \mathrm{mmol}, 50 \mathrm{~mol} \%)$ or diamine iPBP 4c (0.08 mmol, $25 \mathrm{~mol} \%)$ in dry chloroform filtered on basic alumina (3 mL) was added 1,1-bis(benzenesulfonyl)ethylene 2 (0.103 g, $0.335 \mathrm{mmol}, 1$ eq.). Then the aldehyde (3.35 mmol, $10 \mathrm{eq)} \mathrm{was} \mathrm{introduced} \mathrm{at} \mathrm{the} \mathrm{appropriate} \mathrm{temperature.} \mathrm{The} \mathrm{evolution} \mathrm{of}$ the reaction was controlled by TLC until completion. The solution was hydrolysed with $\mathrm{NH}_{4} \mathrm{Cl}$ aq. sat. (2 mL). The layers were separated and the aqueous phase was extracted with $\mathrm{CH}_{2} \mathrm{Cl}_{2}$ (3 x $\left.3 \mathrm{~mL}\right)$. The combined organic phases were dried over $\mathrm{Na}_{2} \mathrm{SO}_{4}$, filtered, concentrated and purified by flash column chromatography on Florisil ${ }^{\circledR}$ using a mixture of cyclohexane (c-Hex) and ethylacetate (AcOEt) as eluent.

\section{Bis(phenylsulfonyl)ethyl)-3-methylbutanal (5a)}

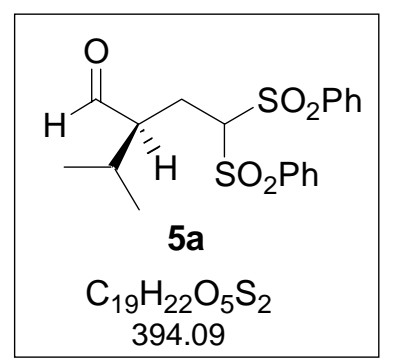

From 3-methyl-butyraldehyde 3a (3.35 mmol, 10 eq., $0.36 \mathrm{~mL}), 1,1-$ bis(benzenesulfonyl)ethylene 2 (0.335 mmol, 1 eq., $0.103 \mathrm{~g})$ and iPBP (0.08 mmol, 0.25 eq., $15 \mathrm{mg}$ ) according to General Procedure 1 (2 hours, $-60^{\circ} \mathrm{C}$ ) to give a yellow oil $(164 \mathrm{mg}$ ) as crude which is purified by column chromatography on Florisil ${ }^{\circledR}$ (c-Hex/AcOEt 2:1) to obtain a pale yellow oil (94 mg, 71\%). The enantiomeric excess was determined by chiral SFC (chiralcel OJ column, $2 \mathrm{~mL} / \mathrm{min}, 200$

\footnotetext{
${ }^{4}$ a) Alexakis, A.; Tomassini, A.; Chouillet, C.; Roland, S.; Mangeney, P.; Bernardinelli, G., Angew. Chem. Int. Ed. 2000, 39, 4093. b) Denmark, S. E.; Fu, J. J. Am. Chem. Soc. 2001, 123, 9488.
} 
bar, MeOH 10\%-2-1-25\%, 30 $\left.{ }^{\circ}, R_{\mathrm{T}}: 4.14(S), 5.80(R)\right) .[\alpha]_{\mathrm{D}}=+36.41\left(\mathrm{CHCl}_{3}\right.$, с $=0.93$ $\mathrm{g} / 100 \mathrm{~mL})$.

${ }^{1} \mathrm{H}$ NMR (400 MHz, $\left.\mathrm{CDCl}_{3}\right): \delta=9.59(\mathrm{~s}, 1 \mathrm{H}), 7.96-7.88\left(\mathrm{dd}, 4 \mathrm{H}, \mathrm{J}_{1}=24.1 \mathrm{~Hz}, \mathrm{~J}_{2}=7.4 \mathrm{~Hz}\right.$ ), 7.73-7.67 (m, 2H), 7.60-7.53 (m, 4H), 4.71-4.68 (dd, 1H, J $\left.=9.1 \mathrm{~Hz}, \mathrm{~J}_{2}=3.1 \mathrm{~Hz}\right), 2.94-2.90$ (m, 1H), 2.54-2.47 (m, 1H), 2.17-2.11 (m, 2H), 0.99 (d, 3H, J= 7.1 Hz), 0.94 (d, 3H, J= 6.8 $\mathrm{Hz})$.

${ }^{13} \mathrm{C}$ NMR (100 MHz, $\left.\mathrm{CDCl}_{3}\right): \delta=203.99$ (1 CHO), 137.89 (1 $\left.\mathrm{C}_{\text {quat. }}\right), 137.74\left(1 \mathrm{C}_{\text {quat. }}\right), 134.75$ (1CH), $134.57(1 \mathrm{CH}), 129.78(1 \mathrm{CH}), 129.37(1 \mathrm{CH}), 129.186(1 \mathrm{CH}), 129.14(1 \mathrm{CH}), 80.55$ $(1 \mathrm{CH}), 54.67(\mathrm{CH}), 28.62(1 \mathrm{CH}), 21.51\left(1 \mathrm{CH}_{2}\right), 19.84\left(1 \mathrm{CH}_{3}\right), 19.04\left(1 \mathrm{CH}_{3}\right)$.

MS (EI mode) m/z (\%) : 396 (1), 225 (28), 169 (12), 145 (14), 143 (25), 141 (11), 134 (13), 125 (49), 97 (15), 91 (17), 83 (19), 81 (10), 79 (12), 78 (25), 77 (100), 69 (13), 67 (10), 55 (35), 51 (35).

IR $\left(\mathrm{CHCl}_{3}\right): 3065 w, 3020 w, 2964 w, 2928 w, 2873 w, 1724 s, 1585 \mathrm{~m}, 1448 \mathrm{~m}, 1331 \mathrm{~s}, 1311 \mathrm{~m}$, $1157 \mathrm{~s}, 1079 \mathrm{~s} \mathrm{~cm}^{-1}$.

HRMS (ESI) calcd for $\mathrm{C}_{19} \mathrm{H}_{22} \mathrm{O}_{5} \mathrm{~S}_{2}[\mathrm{M}+\mathrm{Na}]^{+}$417.08046, found 417.08063.
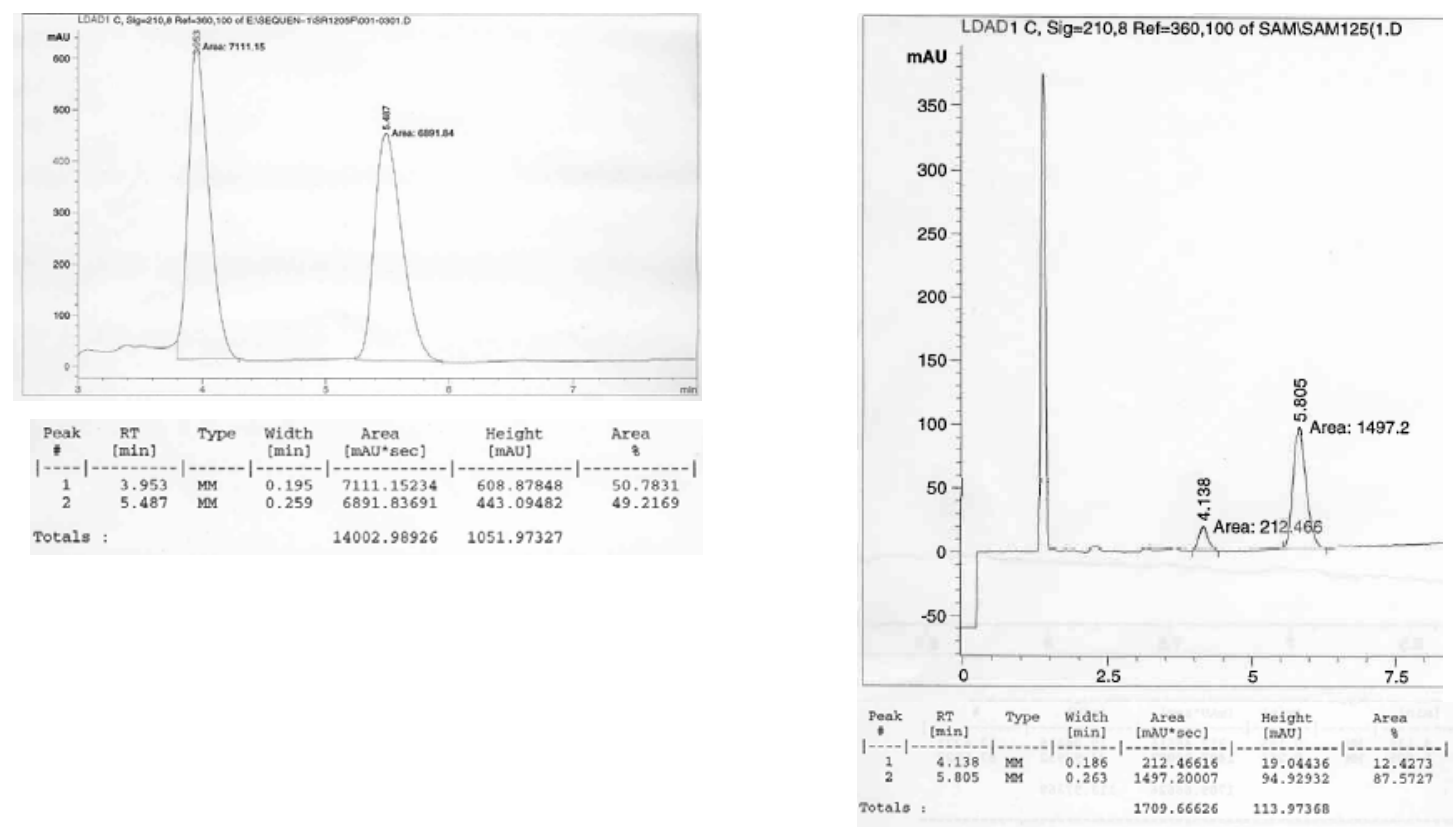

2-(2,2-bis(phenylsulfonyl)ethyl)-3,3-dimethylbutanal (5b)

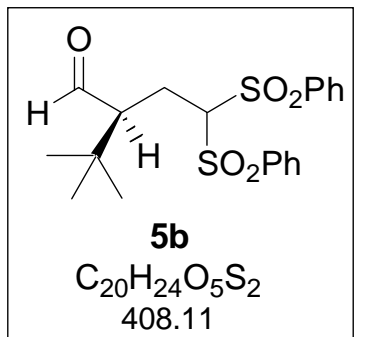

From 3,3-dimethyl butyraldehyde $3 \mathbf{b}(0.41 \mathrm{~mL}, 3.35 \mathrm{mmol}, 10 \mathrm{eq})$, 1,1-bis(benzenesulfonyl)ethylene 2 (0.335 mmol, $1 \mathrm{eq}, 0.103 \mathrm{~g})$ and iPBP (0.08 mmol, 0.25 eq., $15 \mathrm{mg}$ ) according to General Procedure 1 ( 2 hours, $-60^{\circ} \mathrm{C}$ ) to give a yellow oil $(202 \mathrm{mg}$ ) as crude which is purified by column chromatography Florisil ${ }^{\circledR}$ (c-Hex/AcOEt 2:1) to obtain a pale yellow oil (106 mg, 78\%). The enantiomeric excess was determined by SFC (chiralcel OB-H column, $2 \mathrm{~mL} / \mathrm{min}, 200 \mathrm{bar}$,

${ }^{1} \mathrm{H}$ NMR $\left(400 \mathrm{MHz}, \mathrm{CDCl}_{3}\right): \delta=9.71(\mathrm{~s}, 1 \mathrm{H}) ; 7.96-7.86(\mathrm{~m}, 4 \mathrm{H}) ; 7.73-7.68(\mathrm{~m}, 2 \mathrm{H})$; 7.597.53 (m, 4H); 4.57-4.54 (dd, 1H, J= 9.3, 2.5 Hz); 2.79-2.76 (m, 1H); 2.58-2.51 (m, 1H); 2.28$2.21(\mathrm{~m}, 1 \mathrm{H}) ; 1.02(\mathrm{~s}, 9 \mathrm{H})$. 
${ }^{13} \mathrm{C}$ NMR $\left(100 \mathrm{MHz}, \mathrm{CDCl}_{3}\right): \delta=204.91(\mathrm{CHO}), 137.83\left(2 \mathrm{C}_{\text {quat }}\right) ; 134.74(\mathrm{CH}), 134.57(\mathrm{CH})$, $129.73(2 \mathrm{CH}), 129.35(2 \mathrm{CH}), 129.19(2 \mathrm{CH}), 129.14(2 \mathrm{CH}), 80.92(\mathrm{CH}), 58.70(\mathrm{CH}), 33.93$ $\left(\mathrm{C}_{\text {quat }}\right), 27.81\left(3 \mathrm{CH}_{3}\right) ; 20.96\left(\mathrm{CH}_{2}\right)$.

IR ( $\left.\mathrm{CHCl}_{3}\right)$ : 3029 (m), 2967 (s), 2871 (w), 2849 (w), 1716 (s), 1585 (w), 1448 (s), 1373 (m), 1331 (s), 1156 (s), 1079 (s).

MS (EI mode) m/z (\%) : 380 (2), 240 (15), 239 (100), 174 (11), 169 (12), 143 (47), 141 (15), 125 (63), 97m (41), 91 (14), 81 (11), 79 (12), 78 (20), 77 (73), 70 (14), 69 (21), 57 (45), 55 (52), 51 (19).

Microanalysis : calcd for $\mathrm{C}_{20} \mathrm{H}_{24} \mathrm{O}_{5} \mathrm{~S}_{2}$ : C (58.80\%), $\mathrm{H}(5.92 \%)$, found $\mathrm{C}$ (58.80\%), H (5.97\%).
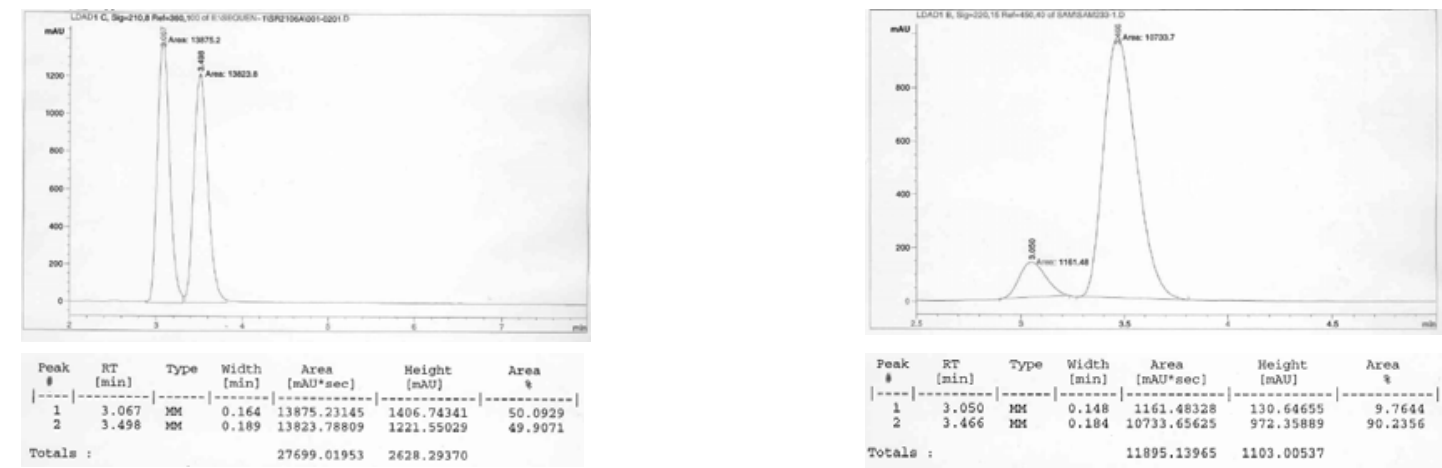

2-cyclohexyl-4,4-bis(phenylsulfonyl)butanal (5c)

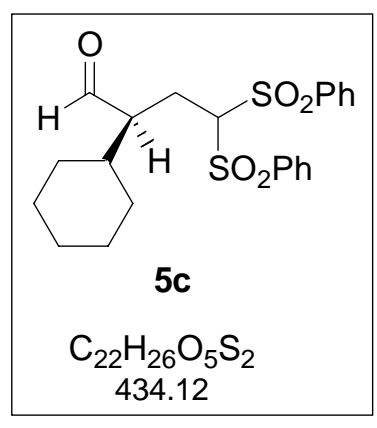

From 2-cyclohexylacetaldehyde 3c (0.422 g, 3.35 mmol, 10 eq.), 1,1bis (benzenesulfonyl) ethylene $2(0.335 \mathrm{mmol}, 1 \mathrm{eq}, 0.103 \mathrm{~g})$ and iPBP ( $0.08 \mathrm{mmol}, 0.25$ eq., $15 \mathrm{mg}$ ) according to General Procedure 1 ( 2 hours, $-60^{\circ} \mathrm{C}$ ) to give a yellow oil $(268 \mathrm{mg}$ ) as crude which is purified by column chromatography Florisil ${ }^{\circledR}$ (c-Hex/AcOEt 2:1) to obtain a pale yellow oil (103 mg, 71\%). The enantiomeric excess was determined by SFC (chiralcel OJ column or chiralpak AS column, 2 $\mathrm{mL} / \mathrm{min}, 200$ bar, $\mathrm{MeOH} 10 \%-2-1-25 \%, 30^{\circ} \mathrm{C}, R_{\mathrm{T}}$ : $\left.4.18(R), 5.08(S)\right)$. $[\alpha]_{\mathrm{D}}=+22.11\left(\mathrm{CHCl}_{3}, \mathrm{c}=1.01 \mathrm{~g} / 100 \mathrm{~mL}\right)$.

${ }^{1} \mathrm{H}$ NMR (400 MHz, $\left.\mathrm{CDCl}_{3}\right): \delta=9.57(\mathrm{~s}, 1 \mathrm{H}) ; 7.95-7.87$ (dd, 4H, J = 22.0, 8.6 Hz); 7.72-7.66 (m, 2H); 7.59-7.54 (m, 4H); 4.67-4.64 (dd, 1H, J= 9.1, 3.3 Hz); 2.91-2.87 (m, 1H); 2.55-2.48 (m, 1H); 2.19-2.12 (m, 1H); 1.76-1.54 (m, 5H), 1.28-1.01 (m, 5H).

${ }^{13} \mathrm{C}$ NMR (100 MHz, $\left.\mathrm{CDCl}_{3}\right): \delta=204.01(\mathrm{CHO}), 137.85\left(\mathrm{C}_{\text {quat }}\right) ; 134.72\left(\mathrm{C}_{\text {quat }}\right), 134.67(\mathrm{CH})$, $134.50(\mathrm{CH}), 129.71(2 \mathrm{CH}), 129.33(2 \mathrm{CH}), 129.13(2 \mathrm{CH}), 129.08(2 \mathrm{CH}), 80.91(\mathrm{CH}), 54.40$ (CH), $38.69(\mathrm{CH}), 30.39\left(\mathrm{CH}_{2}\right), 29.51\left(\mathrm{CH}_{2}\right), 26.33\left(\mathrm{CH}_{2}\right), 26.29\left(\mathrm{CH}_{2}\right), 25.95\left(\mathrm{CH}_{2}\right), 21.74$ $\left(\mathrm{CH}_{2}\right)$.

IR (CHCl $)$ : 2923 (m), 2852 (w), 1721 (m), 1584 (w), 1446 (s), 1325 (s), 1310 (s), 1151 (s), 1142 (s), 1077 (s).

MS (EI mode) m/z (\%) : 435 (<1), 353 (1), 266 (18), 265 (100), 211 (12), 200 (22), 174 (21), 169 (20), 143 (53), 141 (14), 125 (62), 123 (20), 122 (15), 121 (10), 118 (10), 107 (10), 91 (19), 83 (10), 81 (53), 79 (19), 78 (15), 77 (51), 69 (14), 67 (29), 55 (31).

HRMS (ESI) calcd for $\mathrm{C}_{22} \mathrm{H}_{26} \mathrm{O}_{5} \mathrm{~S}_{2}[\mathrm{M}+\mathrm{H}]^{+}$435.1292890, found 435.12929. 

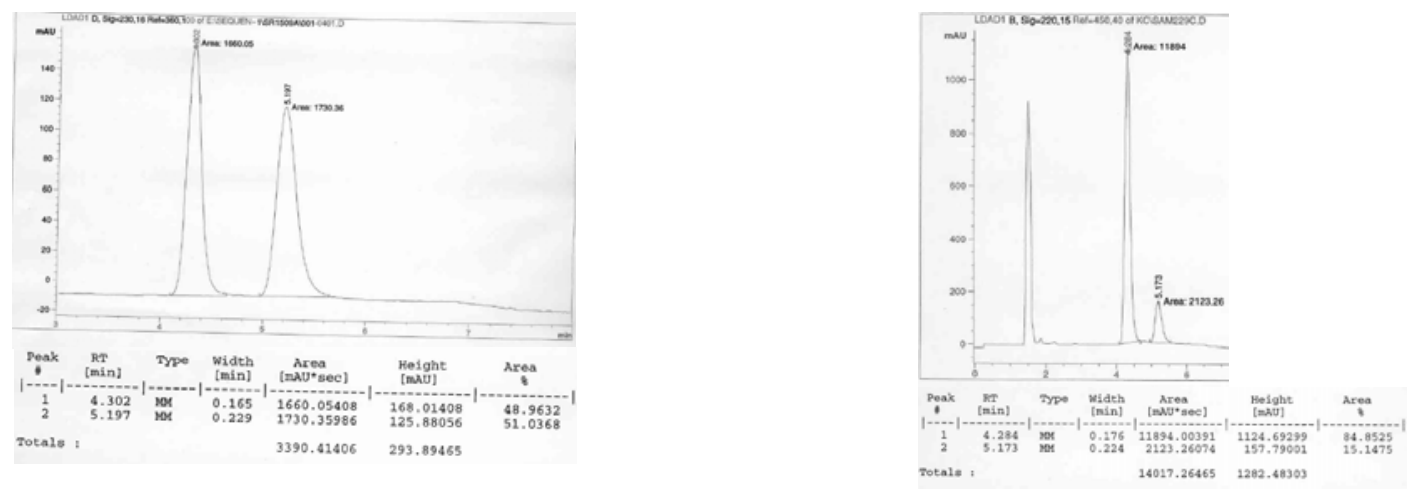

2-(2,2-bis(phenylsulfonyl)ethyl)pentanal (5d)

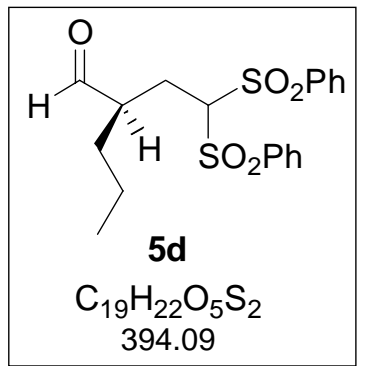

$=1.02 \mathrm{~g} / 100 \mathrm{~mL})$.

${ }^{1} \mathrm{H}$ NMR $\left(400 \mathrm{MHz}, \mathrm{CDCl}_{3}\right): \delta=9.52(\mathrm{~s}, 1 \mathrm{H}), 7.96-7.88\left(\mathrm{dd}, 4 \mathrm{H}, \mathrm{J}_{1}=20.2 \mathrm{~Hz}, \mathrm{~J}_{2}=8 \mathrm{~Hz}\right.$ ), 7.73-7.67 (m, 2H), 7.59-7.54 (m, 4H), 4.74-4.72 (dd, $\left.1 \mathrm{H}, \mathrm{J}_{1}=8 \mathrm{~Hz}, \mathrm{~J}_{2}=4 \mathrm{~Hz}\right), 3.00-2.97(\mathrm{~m}$, $1 \mathrm{H}), 2.51-2.45(\mathrm{~m}, 1 \mathrm{H}), 2.22-2.16(\mathrm{~m}, 1 \mathrm{H}), 1.72-1.66(\mathrm{~m}, 1 \mathrm{H}), 1.45-1.43(\mathrm{~m}, 1 \mathrm{H}), 1.58-1.26$ (m, 2H), 0.94-0.90 (t, 2H, J= 7.2 Hz).

${ }^{13} \mathrm{C}$ NMR (100 MHz, $\left.\mathrm{CDCl}_{3}\right): \delta=203.41$ (1 CHO), 137.75 ( $\left.\mathrm{C}_{\text {quat. }}\right), 137.74\left(\mathrm{C}_{\text {quat. }}\right), 134.71$ $(1 \mathrm{CH}), 134.57(1 \mathrm{CH}), 129.69(2 \mathrm{CH}), 129.43(2 \mathrm{CH}), 129.21(2 \mathrm{CH}), 129.17(2 \mathrm{CH}), 80.60$ $(1 \mathrm{CH}), 48.75(1 \mathrm{CH}), 34.91\left(1 \mathrm{CH}_{2}\right), 26.90\left(1 \mathrm{CH}_{2}\right), 19.74\left(1 \mathrm{CH}_{2}\right), 14.18\left(1 \mathrm{CH}_{3}\right)$.

IR $\left(\mathrm{CHCl}_{3}\right): 3028 \mathrm{w}, 2967 \mathrm{w}, 2929 \mathrm{w}, 2874 \mathrm{w}, 2854 \mathrm{w}, 2726 \mathrm{w}, 1724 \mathrm{~s}, 1584 \mathrm{~m}, 1465 \mathrm{w}, 1448 \mathrm{~m}$, 1331s, $1312 \mathrm{~m}, 1156 \mathrm{~s}, 1079 \mathrm{~s} \mathrm{~cm}^{-1}$.

MS (EI mode) m/z (\%) : 395 (1), 296 (10), 226 (10), 225 (67), 169 (39), 160 (32), 143 (61), 141 (27), 134 (70), 125 (85), 111 (10), 109 (12), 97 (12), 91 (20), 83 (17), 81 (13), 79 (11), 78 (25), 77 (100), 55 (45), 51 (22).
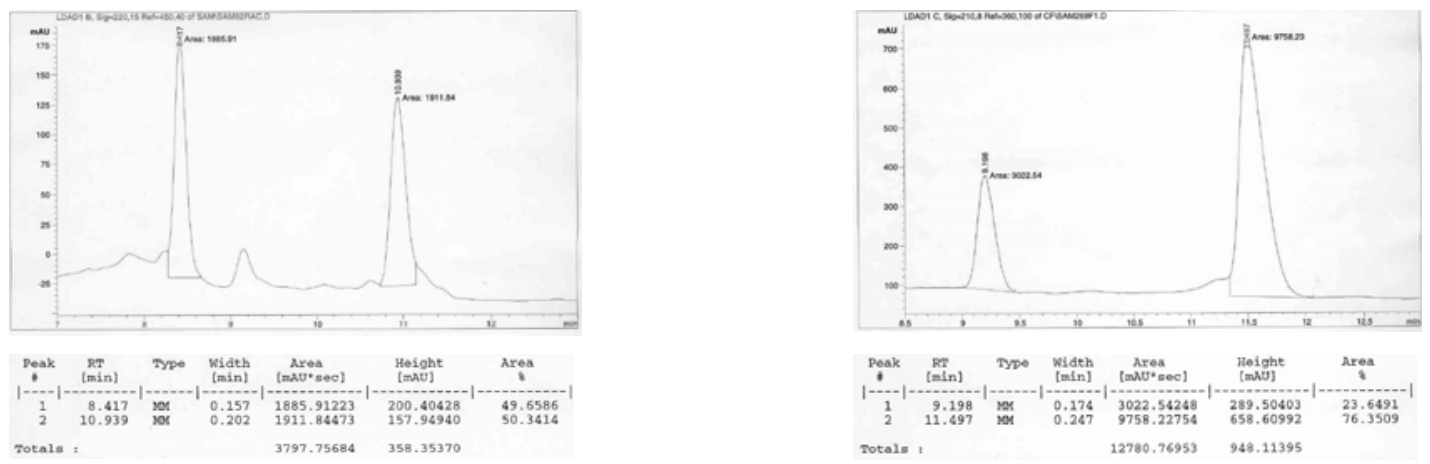


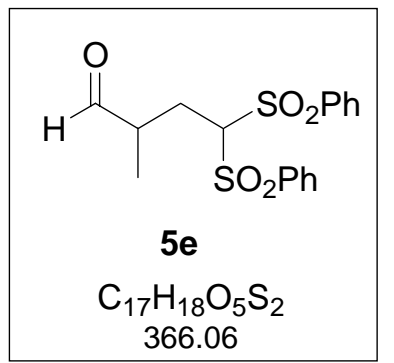

From propionaldehyde 3e $(0.24 \mathrm{~mL}, 3.35 \mathrm{mmol}, 10$ eq. $), 1,1-$ bis(benzenesulfonyl)ethylene 2 (0.335 mmol, $1 \mathrm{eq}, 0.103 \mathrm{~g})$ and pyrrolidine 4a $(0.16 \mathrm{mmol}, 0.5$ eq., $14 \mu \mathrm{L})$ according to General Procedure 1 ( 1 hour, $\left.25^{\circ} \mathrm{C}\right)$ to give a yellow oil $(231 \mathrm{mg})$ as crude which is purified by column chromatography Florisil ${ }^{8}$ (cHex/AcOEt 2:1) to obtain a pale yellow oil (87 mg, 71\%). The enantiomers couldn't be separated by SFC.

${ }^{1} \mathrm{H}$ NMR $\left(400 \mathrm{MHz}, \mathrm{CDCl}_{3}\right): \delta=9.56(\mathrm{~s}, 1 \mathrm{H}) ; 7.95-7.90(\mathrm{~m}, 4 \mathrm{H})$; 7.73-7.69 (m, 2H); 7.60-7.56 (m, 4H); 4.77-4.74 (dd, 1H, J= 7.6, 4.6 Hz); 3.14-3.05 (m, 1H), ; 2.57-2.49 (m, 1H); 2.16-2.08 (m, 1H), 1.17-1.16 (d, 3H, J = 7.3 Hz).

${ }^{13} \mathrm{C}$ NMR $\left(100 \mathrm{MHz}, \mathrm{CDCl}_{3}\right): \delta=202.9(\mathrm{CHO}), 137.68\left(\mathrm{C}_{\text {quat }}\right) ; 137.64\left(\mathrm{C}_{\text {quat }}\right), 134.78(\mathrm{CH})$, $134.67(\mathrm{CH}), 129.70(2 \mathrm{CH}), 129.47(2 \mathrm{CH}), 129.22(2 \mathrm{CH}), 129.18(2 \mathrm{CH}), 80.57(\mathrm{CH}), 43.74$ $(\mathrm{CH}), 26.38\left(\mathrm{CH}_{2}\right), 14.11\left(\mathrm{CH}_{3}\right)$.

IR ( $\left.\mathrm{CHCl}_{3}\right)$ : 2927 (m), 1723 (s), 1584 (w), 1447 (s), 1326 (s), 1311 (s), 1141 (s), 1077 (m).

MS (EI mode) m/z (\%) : 367 (2), 197 (46), 169 (75), 143 (58), 141 (52), 132 (25), 126 (13), 125 (84), 109 (15), 107 (15), 106 (70), 97 (24), 91 (26), 83 (17), 79 (14), 78 (48), 77 (100), 65 (10), 55 (64), 53 (15), 51 (65), 50 (12).

\section{2,2-dimethyl-4,4-bis(phenylsulfonyl)butanal (5f)}

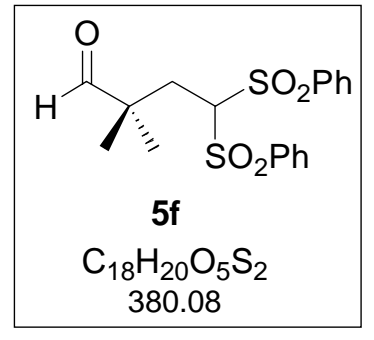

From isobutyraldehyde $3 f$ (3.35 mmol, $10 \mathrm{eq}, 0.30 \mathrm{~mL}$ ), 1,1bis(benzenesulfonyl)ethylene 2 (0.335 mmol, $1 \mathrm{eq}, 0.103 \mathrm{~g})$ and pyrrolidine 4a $(0.16 \mathrm{mmol}, 0.5$ eq., $14 \mu \mathrm{L})$ according to General Procedure 1 ( 1 hour, $\left.25^{\circ} \mathrm{C}\right)$ to give a yellow oil (148 $\left.\mathrm{mg}\right)$ as crude which is purified by column chromatography Florisil ${ }^{\circledR}$ (c-Hex/AcOEt 2:1) to obtain a pale yellow oil (93 mg, 73\%).

${ }^{1} \mathrm{H}$ NMR $\left(400 \mathrm{MHz}, \mathrm{CDCl}_{3}\right): \delta=9.44(\mathrm{~s}, 1 \mathrm{H}), 7.80-7.76(\mathrm{~m}, 4 \mathrm{H})$, 7.63-7.58 (m, 2H), 7.49-7.44 (m, 4H), 4.43 (t, 1H, J=4.3 Hz,), 2.48 (d, 2H, J=4.5 Hz), 1.06 (s, $6 \mathrm{H})$.

${ }^{13} \mathrm{C}$ NMR (100 MHz, $\left.\mathrm{CDCl}_{3}\right): \delta=203.86(1 \mathrm{CHO}), 138.94\left(\mathrm{C}_{\text {quat. }}\right), 136.56(1 \mathrm{CH}), 128.66$ $(1 \mathrm{CH}), 128.16(1 \mathrm{CH}), 79.59(1 \mathrm{CH}), 43.96\left(\mathrm{C}_{\text {quat. }}\right), 30.69\left(1 \mathrm{CH}_{2}\right), 21.35\left(2 \mathrm{CH}_{3}\right)$.

MS (EI mode) m/z (\%) : 381(1), 296 (12), 211 (59), 143 (57), 125 (100), 77 (91), 69 (91).

IR $\left(\mathrm{CHCl}_{3}\right)$ : 2969 (w), 2928 (w), 1721 (s), 1584 (w), 1471 (w), 1448 (s), 1372 (w), 1327 (s), 1312 (s), 1243 (m), 1150 (s), 1079 (s), 1045 (m).

HRMS (ESI) calcd for $\mathrm{C}_{18} \mathrm{H}_{20} \mathrm{O}_{5} \mathrm{~S}_{2}[\mathrm{M}+\mathrm{H}]^{+} 381.4883$, found 381.0819 .

\section{2-(2,2-bis(phenylsulfonyl)ethyl)-2-methylbutanal (5g)}

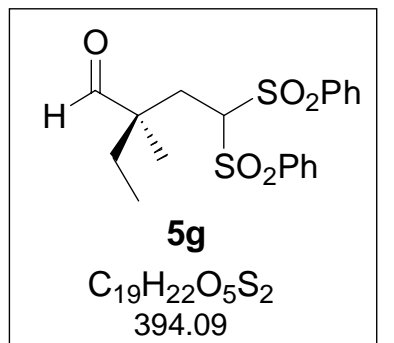

From 2-methyl-butyraldehyde $3 g$ (3.35 mmol, 10 eq, $0.36 \mathrm{~mL}$ ), 1,1bis(benzenesulfonyl)ethylene 2 (0.335 mmol, $1 \mathrm{eq}, 0.103 \mathrm{~g})$ and iPBP (0.08 mmol, 0.25 eq., $15 \mathrm{mg}$ ) according to General Procedure 1 (4 hours, $25^{\circ} \mathrm{C}$ ) to give a yellow oil $(173 \mathrm{mg})$ as crude which is purified by column chromatography Florisil ${ }^{\circledR}$ (c-Hex/AcOEt 2:1) to obtain a pale yellow oil (79 mg, 59\%). The enantiomeric excess was determined by SFC (chiralcel OJ column, $2 \mathrm{~mL} / \mathrm{min}, 200$ bar, $\mathrm{MeOH}$ $\left.10 \%-2-1-25 \%, 30^{\circ} \mathrm{C}, R_{\mathrm{T}}: 5.51(R), 6.28(S)\right) .[\alpha]_{\mathrm{D}}=+70.08\left(\mathrm{CHCl}_{3}, \mathrm{C}\right.$ 
$=1.2 \mathrm{~g} / 100 \mathrm{~mL})$.

${ }^{1} \mathrm{H}$ NMR (400 MHz, $\left.\mathrm{CDCl}_{3}\right): \delta=9.51(\mathrm{~s}, 1 \mathrm{H}), 7.87-7.83(\mathrm{~m}, 4 \mathrm{H}), 7.69-7.65(\mathrm{~m}, 2 \mathrm{H}), 7.55-$ $7.51(\mathrm{~m}, 4 \mathrm{H}), 4.46-4.44$ (dd, $1 \mathrm{H}, \mathrm{J}_{1}=3.7 \mathrm{~Hz}, \mathrm{~J}_{2}=5.1 \mathrm{~Hz}$ ), 2.6-2.54 (dd, $1 \mathrm{H}, \mathrm{J}_{1}=5.1 \mathrm{~Hz}, \mathrm{~J}_{2}=$ $16.4 \mathrm{~Hz}$ ), 2.35-2.30 (dd, $1 \mathrm{H}, \mathrm{J}_{1}=3.5 \mathrm{~Hz}, \mathrm{~J}_{2}=16.4 \mathrm{~Hz}$ ), 1.82-1.73 (q, 1hH, $\mathrm{J}_{1}=7.3 \mathrm{~Hz}, \mathrm{~J}_{2}=6.8$ $\mathrm{Hz}$ ), 1.51-1.44 (q, 1H, J $\mathrm{J}_{1}=7.3 \mathrm{~Hz}, \mathrm{~J}_{2}=6.8 \mathrm{~Hz}$ ), 0.95 (s, 3H), 0.85 (t, 3H, J = 7.3).

${ }^{13} \mathrm{C}$ NMR (100 MHz, $\left.\mathrm{CDCl}_{3}\right): \delta=205.95$ (1 CHO), $137.9\left(\mathrm{C}_{\text {quat. }}\right), 137.36(1 \mathrm{CH}), 134.71$ $(1 \mathrm{CH}), 134.56(1 \mathrm{CH}), 79.40(1 \mathrm{CH}), 48.39\left(\mathrm{C}_{\text {quat. }}\right), 30.64\left(1 \mathrm{CH}_{2}\right), 29.47\left(\mathrm{CH}_{2}\right), 17.05\left(1 \mathrm{CH}_{3}\right)$, $8.37\left(1 \mathrm{CH}_{3}\right)$.

IR ( $\left.\mathrm{CHCl}_{3}\right)$ :3029 (w), $2972(\mathrm{w}), 2929$ (w), 1718 (s), 1585 (w), 1459 (w), 1448 (s), 1331 (s), 1316 (s), 1155 (s), 1079 (s).

MS (EI mode) m/z (\%) : 395 (1), 347 (1), 296 (10), 226 (10), 225 (65), 223 (18), 169 (11), 143 (54), 141 (17), 134 (37), 126 (10), 125 (86), 97 (16), 91 (25), 83 (71), 82 (36), 81 (24), 79 (16), 78 (27), 77 (100), 67 (24), 55 (64), 53 (14), 51 (32).

Microanalysis : calcd for $\mathrm{C}_{19} \mathrm{H}_{22} \mathrm{O}_{5} \mathrm{~S}_{2}$ : C (57.85\%), H (5.62\%), found C (58.06\%), H (5.86\%).
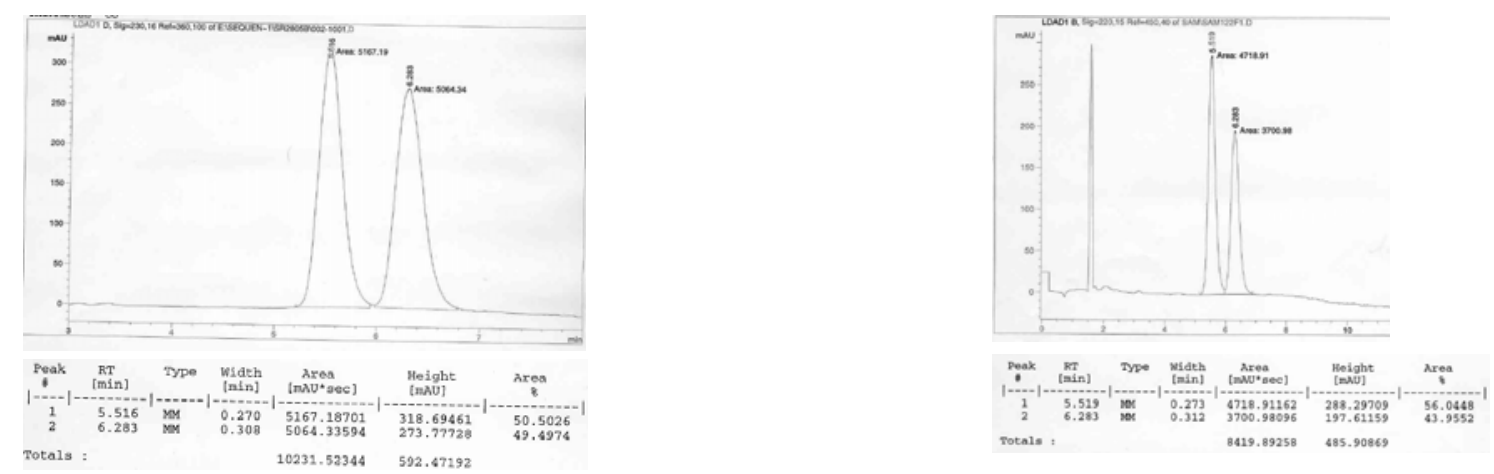

2-methyl-2-phenyl-4,4-bis(phenylsulfonyl)butanal (5h)

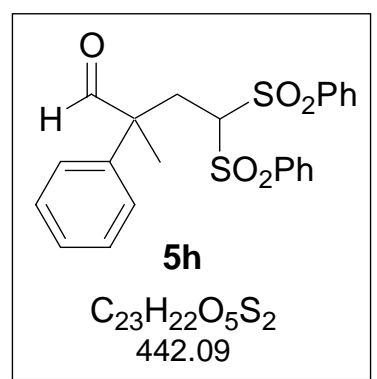

From 2-phenylpropionaldehyde $3 \mathbf{h}$ (0.44 mL, 3.35 mmol, 10 eq.), 1,1bis(benzenesulfonyl)ethylene 2 ( $0.335 \mathrm{mmol}, 1 \mathrm{eq}, 0.103 \mathrm{~g})$ and $\mathbf{i P B P}$ (0.08 mmol, 0.25 eq., $15 \mathrm{mg}$ ) according to General Procedure 1 (7 hours, $25^{\circ} \mathrm{C}$ ) to give a yellow oil (452 $\mathrm{mg}$ ) as crude which is purified by column chromatography Florisil ${ }^{\circledR}$ (c-Hex/AcOEt 2:1) to obtain a pale yellow oil (10 mg, 14\%). The enantiomeric excess was determined by SFC (chiralcel OJ column or chiralcel OB-H column, 2 $\mathrm{mL} / \mathrm{min}, 200$ bar, $\mathrm{MeOH} 10 \%-2-1-25 \%, 30^{\circ} \mathrm{C}, R_{\mathrm{T}}$ : 5.03, 6.84).

${ }^{1} \mathrm{H}$ NMR $\left(400 \mathrm{MHz}, \mathrm{CDCl}_{3}\right): \delta=9.60(\mathrm{~s}, 1 \mathrm{H}) ; 7.88-7.85(\mathrm{~m}, 2 \mathrm{H}) ; 7.68-7.41(\mathrm{~m}, 12 \mathrm{H})$; 7.287.27 (m, 1H); 4.42-4.40 (dd, 1H, J= 8.1, 3.1 Hz); 2.96-2.92 (dd, $1 \mathrm{H}, \mathrm{J}=16,3.1 \mathrm{~Hz})$, ; $2.84-$ 2.80 (dd, $1 \mathrm{H}, \mathrm{J}=17.7,4.6 \mathrm{~Hz}) ; 1.46$ (s, 3H).

${ }^{13} \mathrm{C}$ NMR (100 MHz, $\left.\mathrm{CDCl}_{3}\right): \delta=201.32(\mathrm{CHO}), 138.35\left(\mathrm{C}_{\text {quat }}\right) ; 137.85\left(\mathrm{C}_{\text {quat }}\right), 137.40$ (C $\left.\mathrm{C}_{\text {quat }}\right), 134.54(\mathrm{CH}), 134.52(\mathrm{CH}), 129.80(2 \mathrm{CH}), 129.76(2 \mathrm{CH}), 129.25(2 \mathrm{CH}), 129.05$ $(2 \mathrm{CH}), 129.04(2 \mathrm{CH}), 128.09(\mathrm{CH}), 127.61(2 \mathrm{CH}), 80.24(\mathrm{CH}), 53.10\left(\mathrm{C}_{\text {quat }}\right), 30.91\left(\mathrm{CH}_{2}\right)$, $19.60\left(\mathrm{CH}_{3}\right)$.

IR $\left(\mathrm{CHCl}_{3}\right)$ : 3029 (m), 1723 (s), 1600 (w), 1448 (s), 1331 (s), 1313 (s), 1158 (s), 1079 (m). MS (EI mode) m/z (\%) : 443 (<1), 395 (1), 273 (18), 143 (12), 134 (11), 131 (100), 130 (23), 129 (84), 125 (24), 121 (15), 119 (29), 105 (41), 103 (26), 91 (30), 79 (10), 78 (14), 77 (72), $51(15)$.

HRMS (ESI) calcd for $\mathrm{C}_{23} \mathrm{H}_{22} \mathrm{O}_{5} \mathrm{~S}_{2}[\mathrm{M}+\mathrm{H}]^{+}$443.0990760, found 443.09908. 


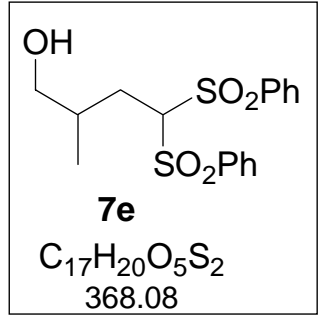

From propionaldehyde $3 \mathbf{e}(0.24 \mathrm{~mL}, 3.35 \mathrm{mmol}, 10$ eq. $)$, 1,1bis(benzenesulfonyl)ethylene 2 (0.335 mmol, $1 \mathrm{eq}, 0.103 \mathrm{~g})$ and iPBP (0.08 mmol, 0.25 eq., $15 \mathrm{mg}$ ) according to General Procedure 1 (2 hours, $\left.-60^{\circ} \mathrm{C}\right)$ to give a yellow oil $(173 \mathrm{mg})$ as crude. Then, it was diluted in $\mathrm{MeOH}(3 \mathrm{~mL})$, cooled to $0^{\circ} \mathrm{C}$, and $\mathrm{NaBH}_{4}$ was added by portion (17 mg, $0.435 \mathrm{mmol}, 1.3 \mathrm{eq}$.). After $1 \mathrm{~h}$ at $0^{\circ} \mathrm{C}$, the conversion was completed. The solution was hydrolysed with $\mathrm{NH}_{4} \mathrm{Cl}$ aq. sat. (3 mL).

The layers were separated and the aqueous phase was extracted with EtOAc ( $2 \mathrm{x} 4 \mathrm{~mL})$. The combined organic phases were dried over $\mathrm{Na}_{2} \mathrm{SO}_{4}$, filtered, concentrated and purified by flash column chromatography on silica gel (c-Hex/EtOAc 1:1) to obtain a yellow pale oil 7e (89 mg, 72\%). The enantiomeric excess was determined by SFC (chiralpak AD column, 2 $\mathrm{mL} / \mathrm{min}, 200$ bar, MeOH 10\%-2-1-25\%, 30 ${ }^{\circ} \mathrm{C}, R_{\mathrm{T}}$ : 8.85, 9.75)

${ }^{1} \mathrm{H}$ NMR (400 MHz, $\left.\mathrm{CDCl}_{3}\right): \delta=8.01-7.97(\mathrm{~m}, 4 \mathrm{H}) ; 7.76-7.72(\mathrm{~m}, 2 \mathrm{H}) ; 7.64-7.60(\mathrm{~m}, 4 \mathrm{H})$; 5.02-4.99(t, 1H, J= 5.56 Hz); 3.67-3.64 (m, 1H), ; 3.45-3.41 (m, 1H); 2.37-2.30 (m, 1H), 2.15$2.10(\mathrm{~m}, 1 \mathrm{H}), 1.57-1.50(\mathrm{~m}, 2 \mathrm{H}), 0.89-0.87$ (d, J=6.56 Hz, 3H).

${ }^{13} \mathrm{C}$ NMR $\left(100 \mathrm{MHz}, \mathrm{CDCl}_{3}\right): \delta=138.00\left(\mathrm{C}_{\text {quat }}\right) ; 137.76\left(\mathrm{C}_{\text {quat }}\right), 134.81(\mathrm{CH}), 129.78(\mathrm{CH})$, $129.97(2 \mathrm{CH}), 129.79(2 \mathrm{CH}), 129.33(2 \mathrm{CH}), 129.27(2 \mathrm{CH}), 81.72(\mathrm{CH}), 68.04\left(\mathrm{CH}_{2}\right), 34.38$ $(\mathrm{CH}), 30.16\left(\mathrm{CH}_{2}\right), 17.00\left(\mathrm{CH}_{3}\right)$.

IR : 3540 (br OH), 2964 (m), 2929 (m), 2876 (m), 1732 (m), 1584(w), 1447 (s), 1326 (s), $1310 \quad(\mathrm{~s}), \quad 1244 \quad(\mathrm{~m}), \quad 1144 \quad(\mathrm{~s}), \quad 1078$ (s), 1043 (s), $731 \quad$ (s), $686 \quad$ (s). MS (EI mode) m/z (\%) : 338 (3), 209 (30), 197 (14), 169 (57), 143 (31), 141 (26), 132 (14), 125 (67), 106 (29), 97 (11), 85 (100), 78 (25), 77 (91), 69 (15), 67 (15), 59 (13), 57 (18), 55 (30), 51 (23).

HRMS (ESI) calcd $\mathrm{C}_{17} \mathrm{H}_{21} \mathrm{O}_{5} \mathrm{~S}_{2}[\mathrm{M}+\mathrm{H}]^{+}$369.0824, found 369.0847 . 


\section{Determination of absolute configuration of 1,4-adducts :}

\section{2-(2,2-bis(phenylsulfonyl)ethyl)-3-methylbutan-1-ol (7a)}

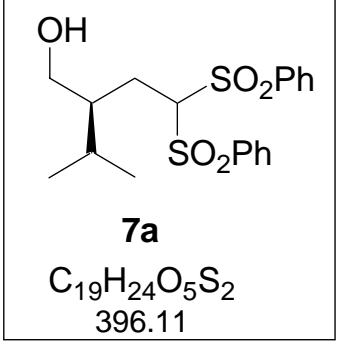

EtOAc $(2 \times 3 \mathrm{~mL})$. The combined organic phases were dried over $\mathrm{Na}_{2} \mathrm{SO}_{4}$, filtered, concentrated and purified by flash column chromatography on silica gel (c-Hex/EtOAc 1:1) to obtain a yellow pale oil 7a (275 mg, 69\%). The enantiomeric excess was determined by SFC (chiralcel OJ column, $2 \mathrm{~mL} / \mathrm{min}, 200$ bar, $\mathrm{MeOH} 10 \%-2-1-25 \%, 30^{\circ} \mathrm{C}, R_{\mathrm{T}}: 5.28(R), 5.84(S)$ ). $[\alpha]_{D}=-19.82\left(\mathrm{CHCl}_{3}, \mathrm{c}=1.025 \mathrm{~g} / 100 \mathrm{~mL}\right)$.

${ }^{1} \mathrm{H}$ NMR (400 MHz, $\left.\mathrm{CDCl}_{3}\right): \delta=7.97-7.93(\mathrm{~m}, 4 \mathrm{H}) ; 7.69-7.67(\mathrm{~m}, 2 \mathrm{H}) ; 7.59-7.55(\mathrm{~m}, 4 \mathrm{H})$; 5.22-5.20 (dd, 1H, J= 7.3, 3.3 Hz); 3.75-3.71 (m, 1H), ; 3.54-3.49 (m, 1H); 2.33-2.26 (m, 1H), 2.22-2.16 (m, 1H), 1.76-1.60 (m, 2H), 0.85-0.82 (m, 6H).

${ }^{13} \mathrm{C}$ NMR $\left(100 \mathrm{MHz}, \mathrm{CDCl}_{3}\right): \delta=137.96\left(\mathrm{C}_{\text {quat }}\right) ; 137.73\left(\mathrm{C}_{\text {quat }}\right), 134.55\left(\mathrm{C}_{\text {quat }}\right), 134.52(\mathrm{CH})$, $129.72(2 \mathrm{CH}), 129.59(2 \mathrm{CH}), 129.12(2 \mathrm{CH}), 129.05(2 \mathrm{CH}), 81.63(\mathrm{CH}), 64.80\left(\mathrm{CH}_{2}\right), 44.46$ $(\mathrm{CH}), 29.84(\mathrm{CH}), 26.14\left(\mathrm{CH}_{2}\right), 19.47\left(\mathrm{CH}_{3}\right), 19.27\left(\mathrm{CH}_{3}\right)$.

IR : 3545 (br OH), 2958 (m), 2928 (m), 1732 (m), 1584(w), 1447 (s), 1326 (s), 1310 (s), 1244 (m), 1144 (s), 1078 (s), 731 (s), 686 (s).

MS (EI mode) m/z (\%) : 366 (3), 237 (29), 225 (51), 169 (36), 160 (25), 149 (15), 143 (44), 141 (17), 134 (23), 125 (69), 113 (41), 97 (17), 95 (59), 94 (19), 91 (13), 83 (21), 81 (14), 79 (20), 78 (31), 77 (100), 71 (15), 70 (10), 69 (23), 67 (15), 57 (11), 55 (38), 53 (12), 51 (28).

HRMS (ESI) calcd $\mathrm{C}_{19} \mathrm{H}_{24} \mathrm{O}_{5} \mathrm{~S}_{2}[\mathrm{M}+\mathrm{Na}]^{+}$396.10652, found 419.09563.
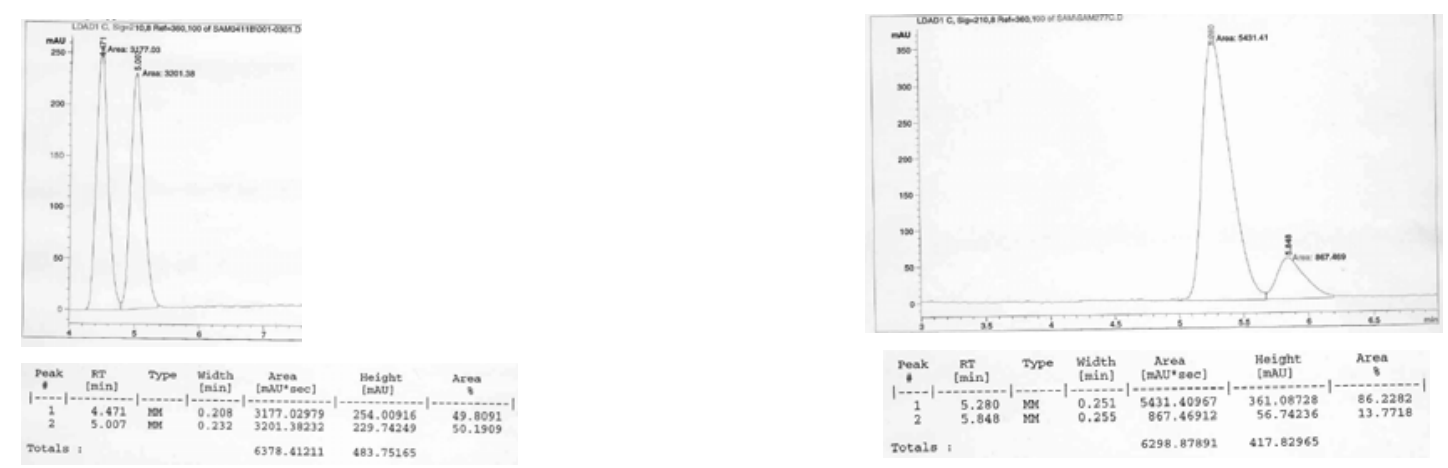

(S)-2-ethyl-3-methyl-1-butanol (8)

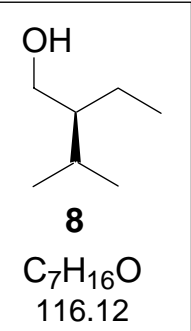

To Mg turnings (0.568 g, 23.4 mmol, 45 eq.) activated by TMSCl (1 drop) and 1,2-dibromoethane (1 drop) was added dropwise a solution of alcohol 7a (206 mg, $0.52 \mathrm{mmol}, 1$ eq.) in $\mathrm{MeOH}(8 \mathrm{~mL})$ at $0^{\circ} \mathrm{C}$. After $1 \mathrm{~h}$ at $0^{\circ} \mathrm{C}$, the reaction mixture was allowed to reflux overnight. Then, after cooling at room temperature, it was diluted in $\mathrm{Et}_{2} \mathrm{O}(10 \mathrm{~mL})$ and hydrolyzed with $\mathrm{HCl} 1 \mathrm{~N}$ (10 $\mathrm{mL}$ ). The layers were separated and the aqueous phase was extracted with $\mathrm{Et}_{2} \mathrm{O}$ 
(4 x $10 \mathrm{~mL}$ ). The combined organic phases were washed by $\mathrm{H}_{2} \mathrm{O}$ and dried over $\mathrm{Na}_{2} \mathrm{SO}_{4}$, filtered, concentrated and purified by flash column chromatography on silica gel $\left(\mathrm{Et}_{2} \mathrm{O} /\right.$ pentane $\left.4: 1\right)$ to obtain a yellow pale oil 8 (27 mg, 45\%). The enantiomeric excess was determined by GC (CHIRASIL-DEX-CB column : 50 C-0-1-170-0, $R_{\mathrm{T}}$ : $37.97(\mathrm{~S}), 38.73(\mathrm{R})$ ). $[\alpha]^{25}=-6.79\left(\mathrm{CHCl}_{3}, \quad \mathrm{c}=0.118 \mathrm{~g} / 100 \mathrm{~mL}\right)$ compared to literature data ${ }^{5}[\alpha]^{20}=$ 9.47( $\left.c=2.126, \mathrm{CHCl}_{3}\right)$.

GC/MS (EI) (50 C-2-10-270-5) : $\mathrm{R}_{\mathrm{T}}=6.27$ min (100\%) : 98 (10), 85 (100), 83 (15), 69 (55), $56(60)$.

${ }^{1} \mathrm{H}$ NMR (400 MHz, $\left.\mathrm{CDCl}_{3}\right): \delta=3.66-3.56(\mathrm{~m}, 2 \mathrm{H}), 1.84-1.76(\mathrm{~m}, 1 \mathrm{H}), 1.44-1.45(\mathrm{~m}, 1 \mathrm{H})$, 1.23-1.25 (m, 2H), 0.93-0.88 (m, 9H).

Spectroscopic data are in agreement with published data. ${ }^{5}$

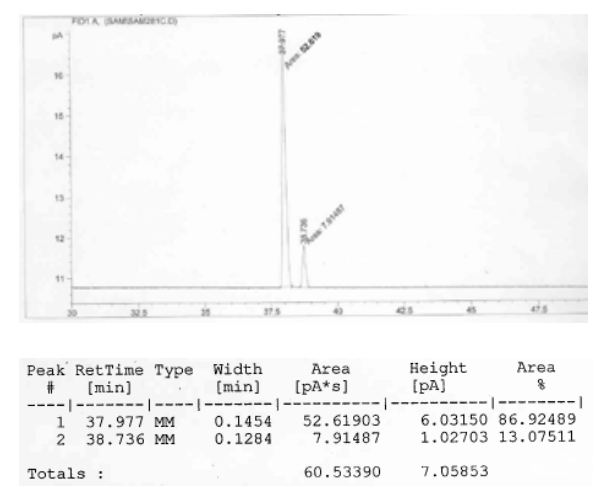

${ }^{5}$ (a) Gao, L-J.; Zhao, X-Y.; Vandewalle, M.; De Clercq, P. Eur. J. Org. Chem. 2000, 2755. (b) Tsuda, K.; Kishida, Y.; Hayatsu, R. J. Am. Chem. Soc. 1960, 82, 3396. 


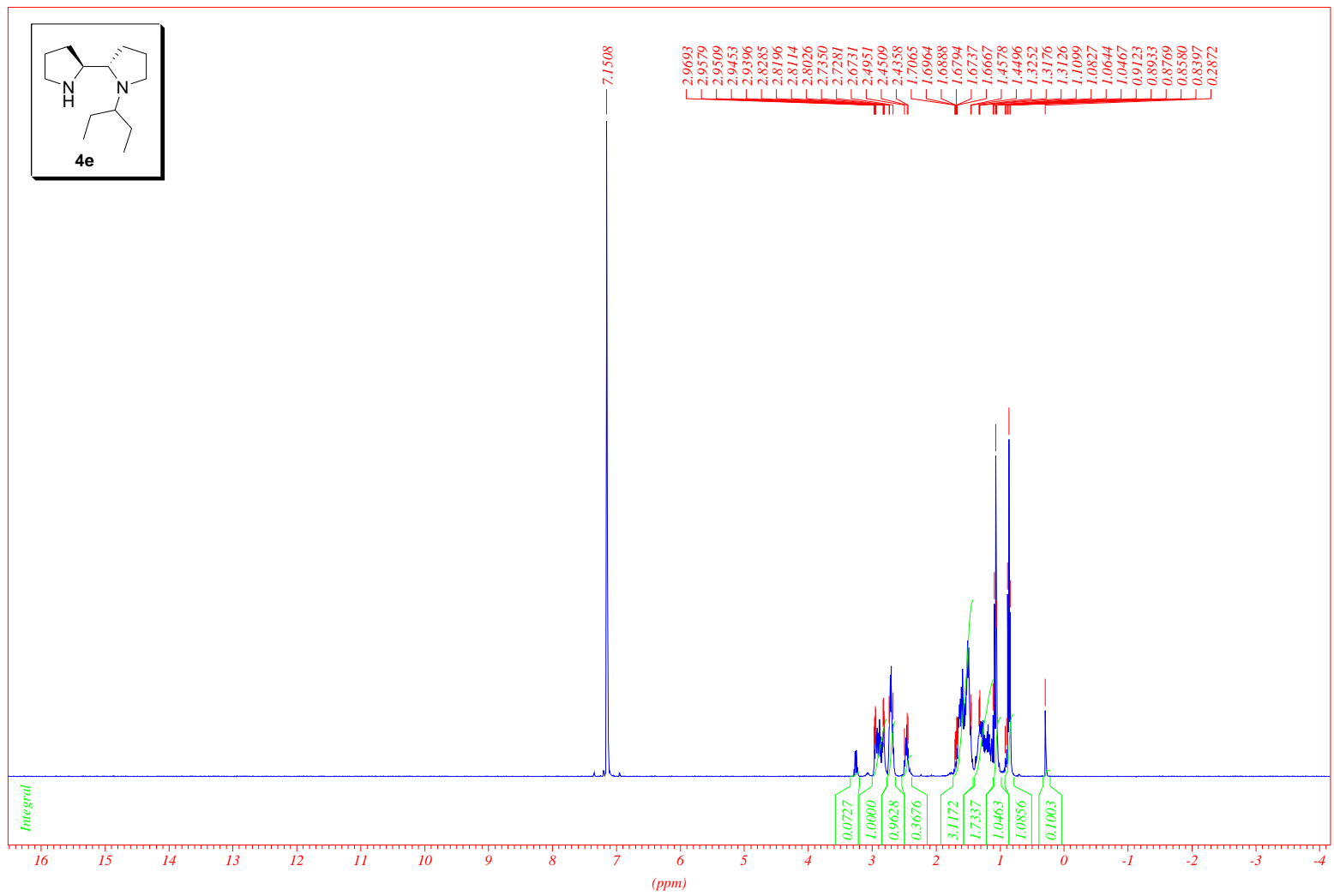

$13 \mathrm{C}$ (C6D6, $100 \mathrm{MHz}$ )

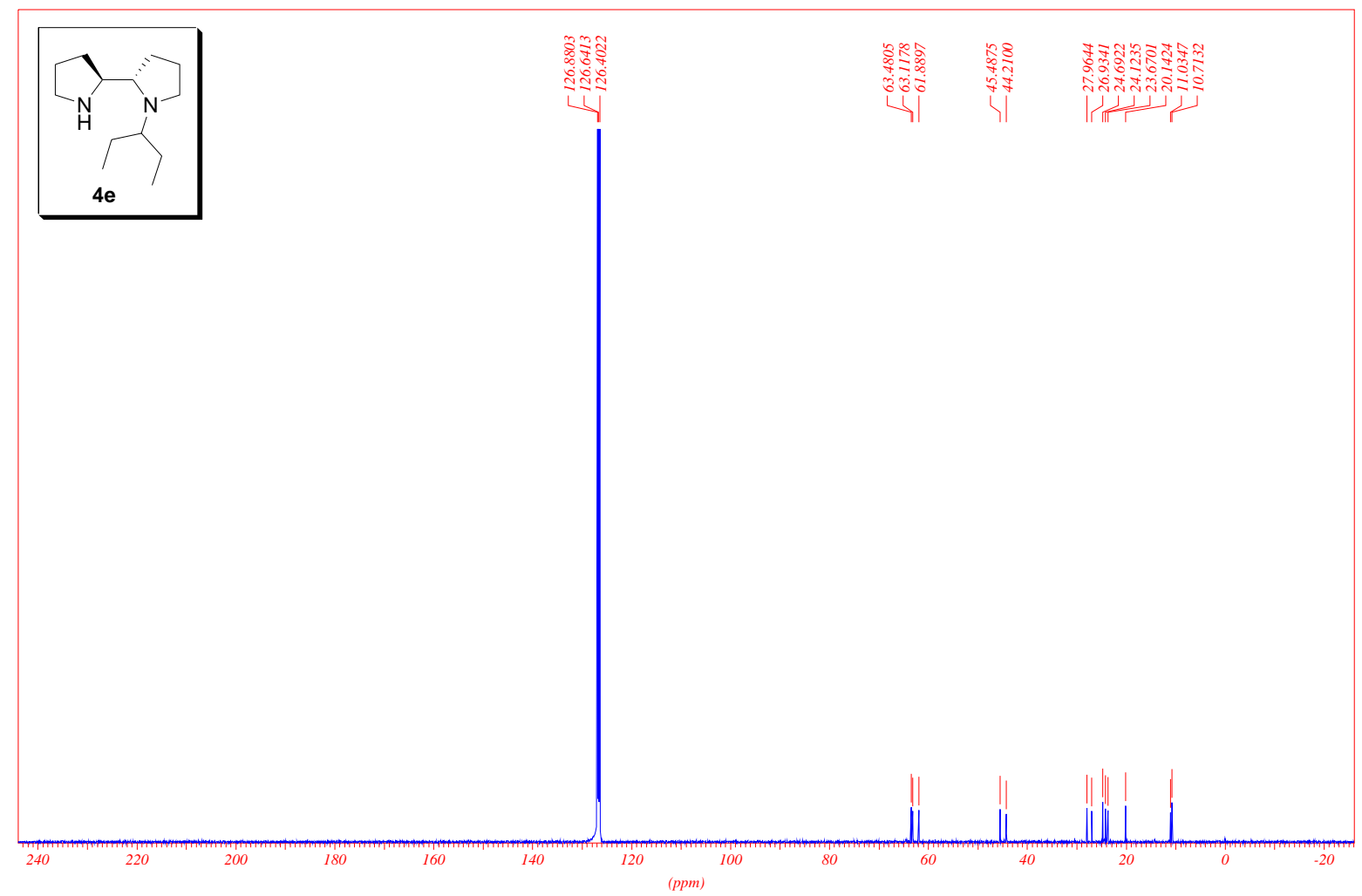




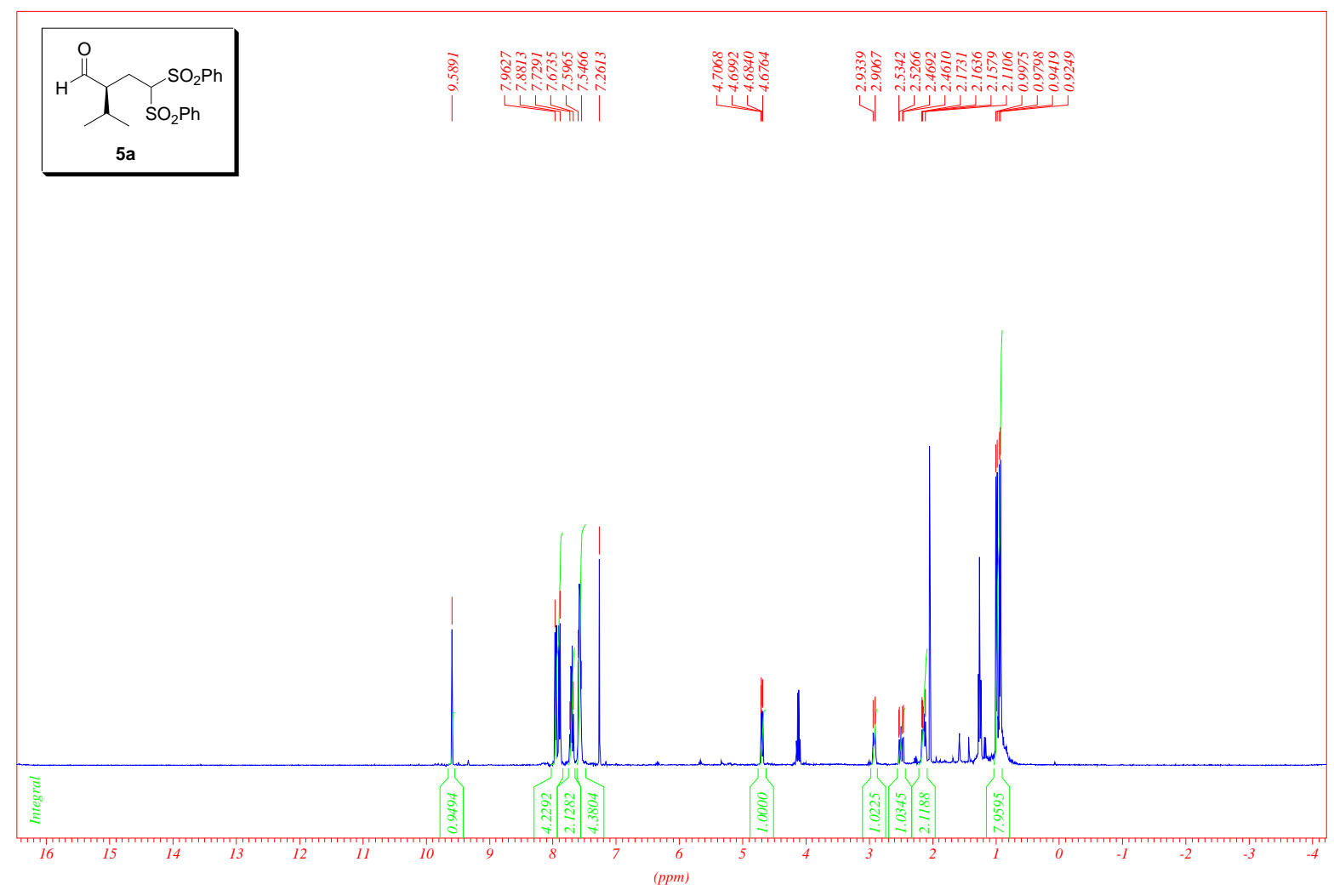

13C (CDCl3, $100 \mathrm{MHz})$

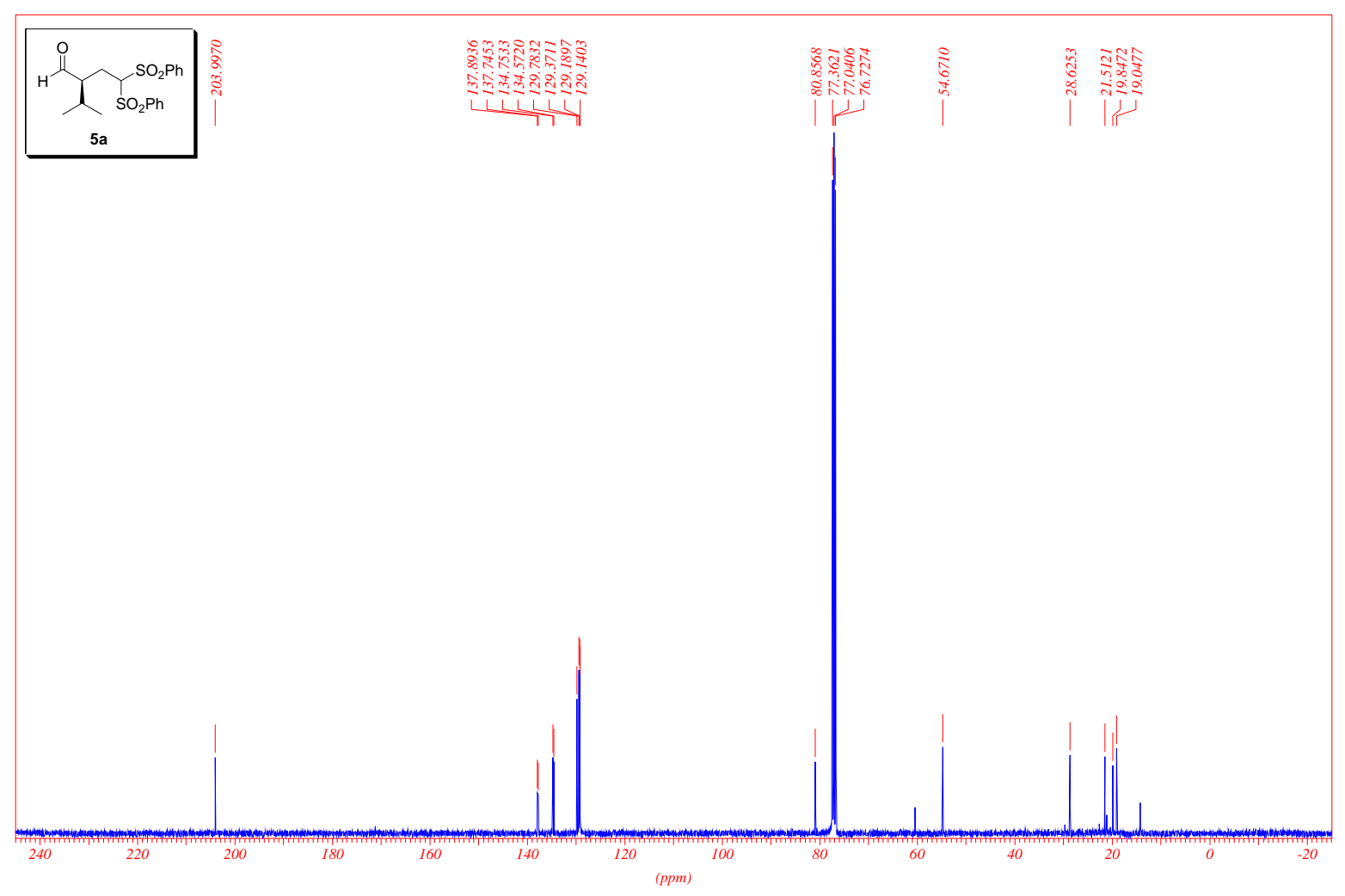




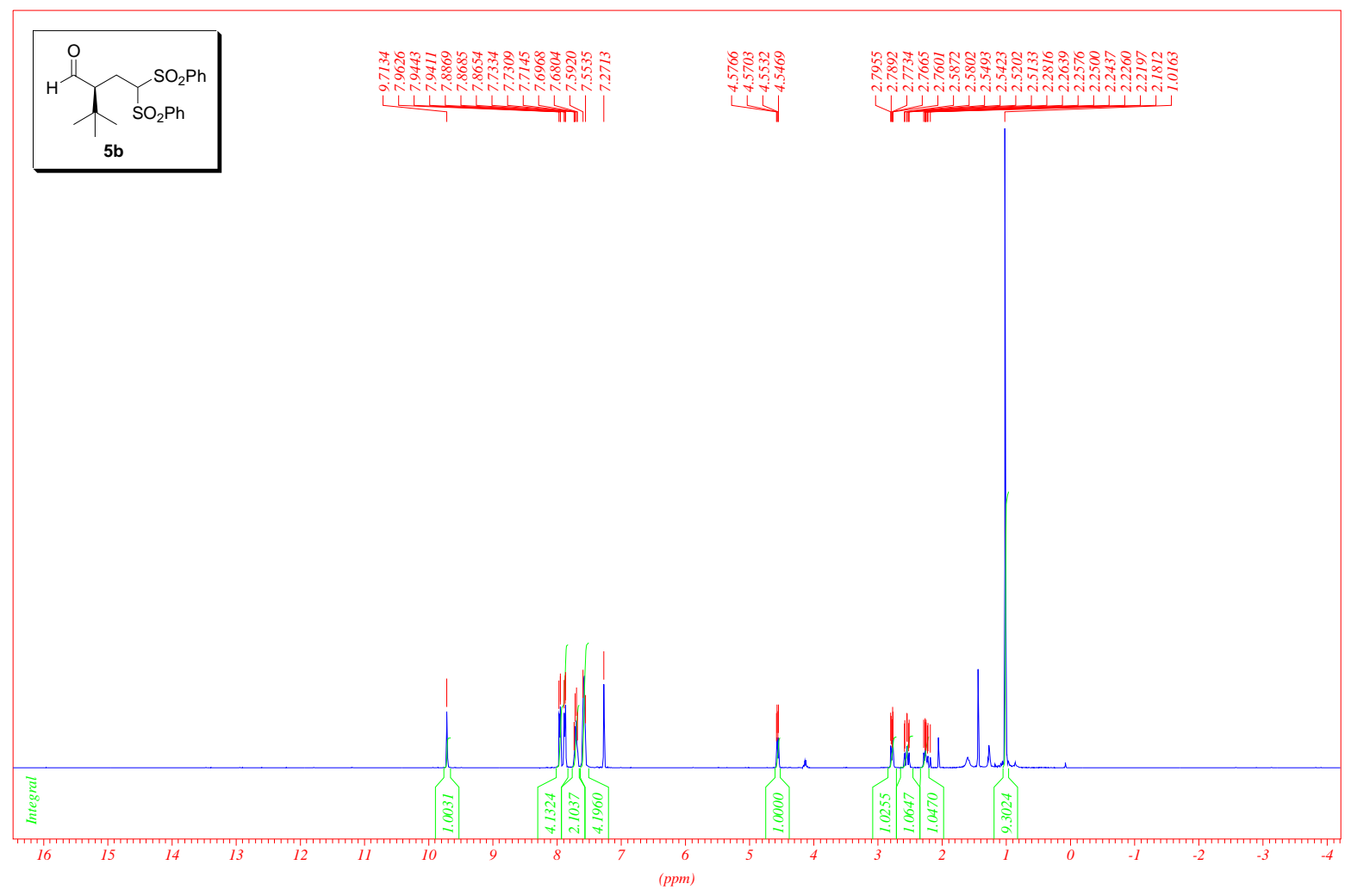

$13 \mathrm{C}(\mathrm{CDCl} 3,100 \mathrm{MHz})$

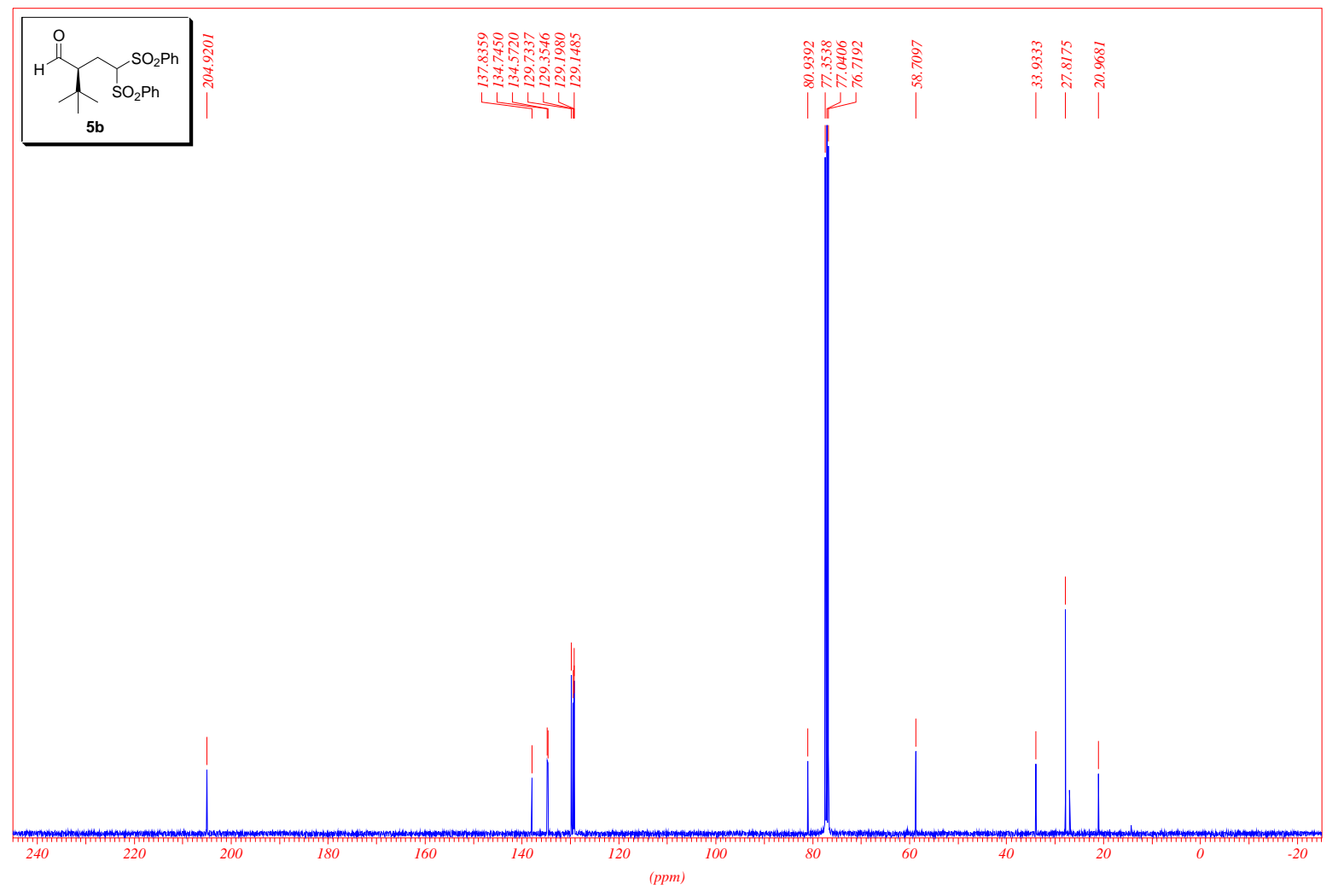




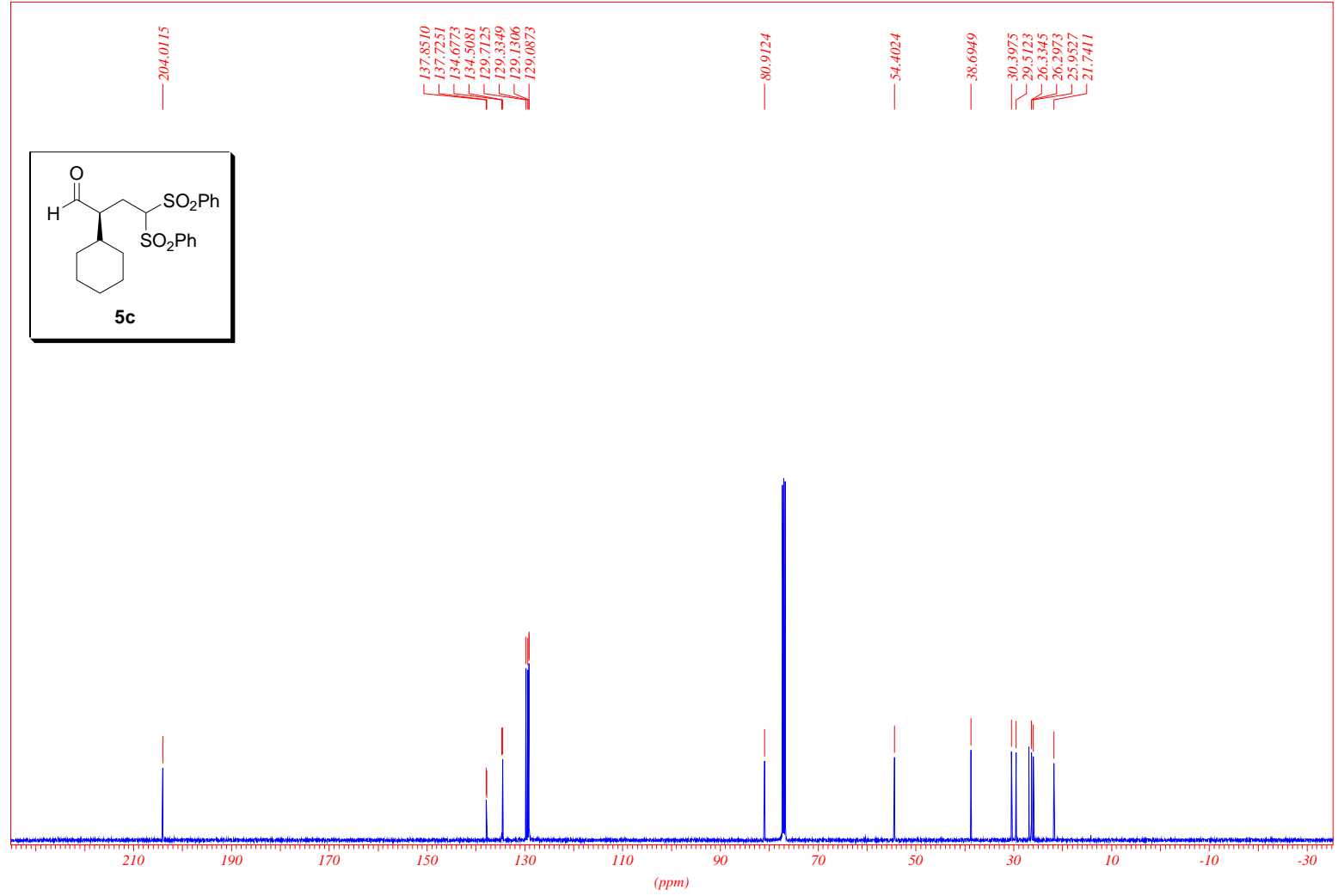

$1 \mathrm{H}(\mathrm{CDCl} 3,400 \mathrm{MHz})$

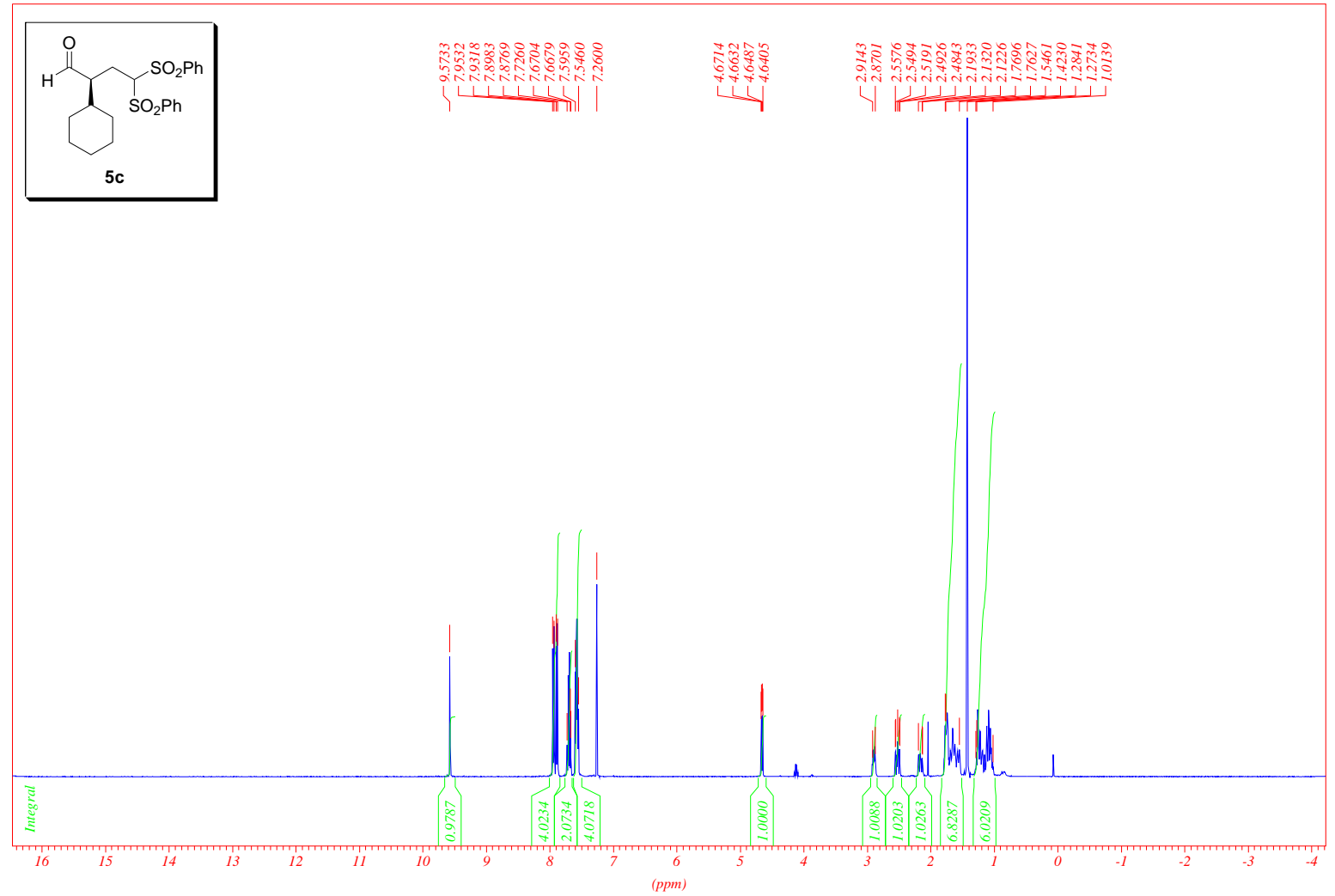



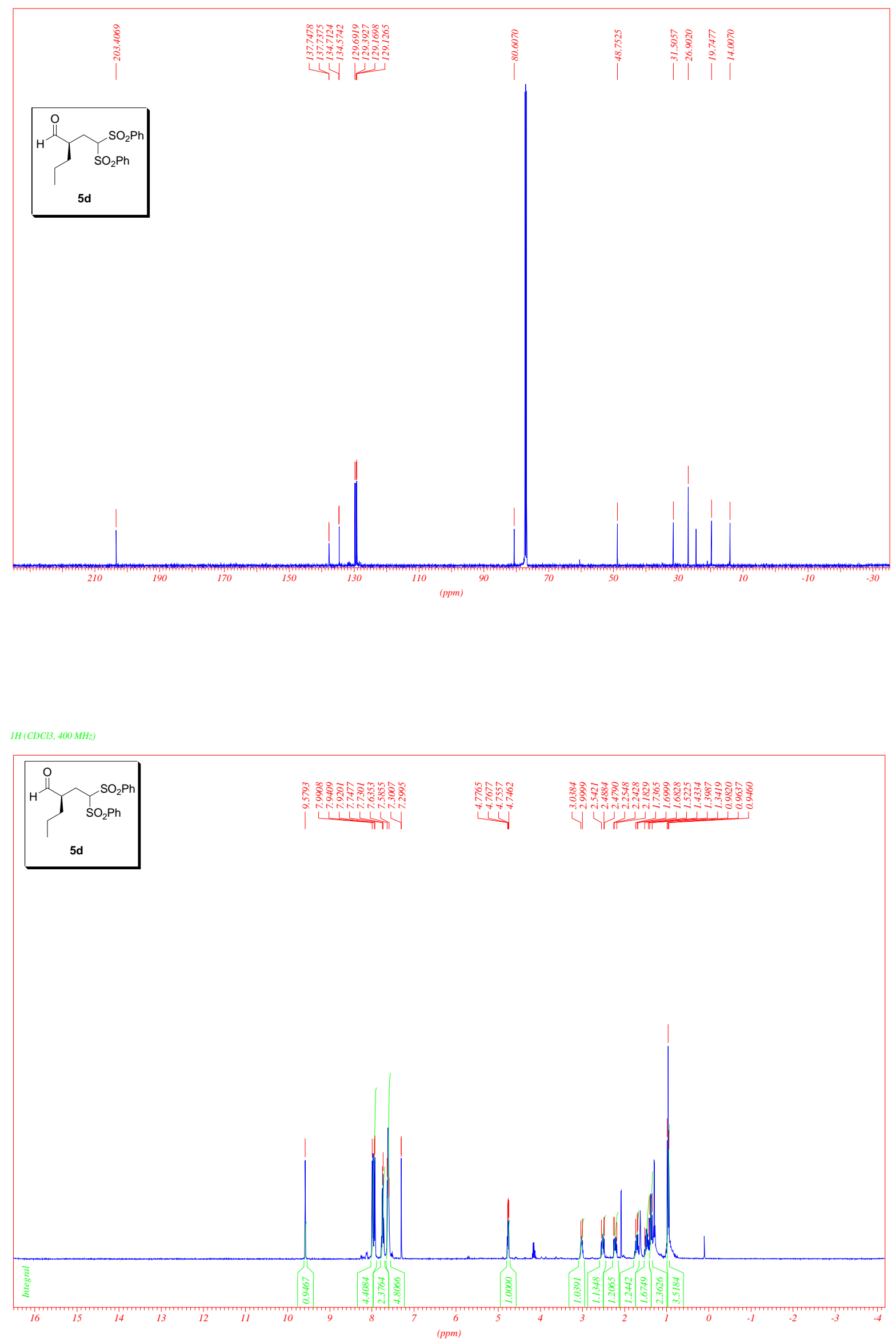


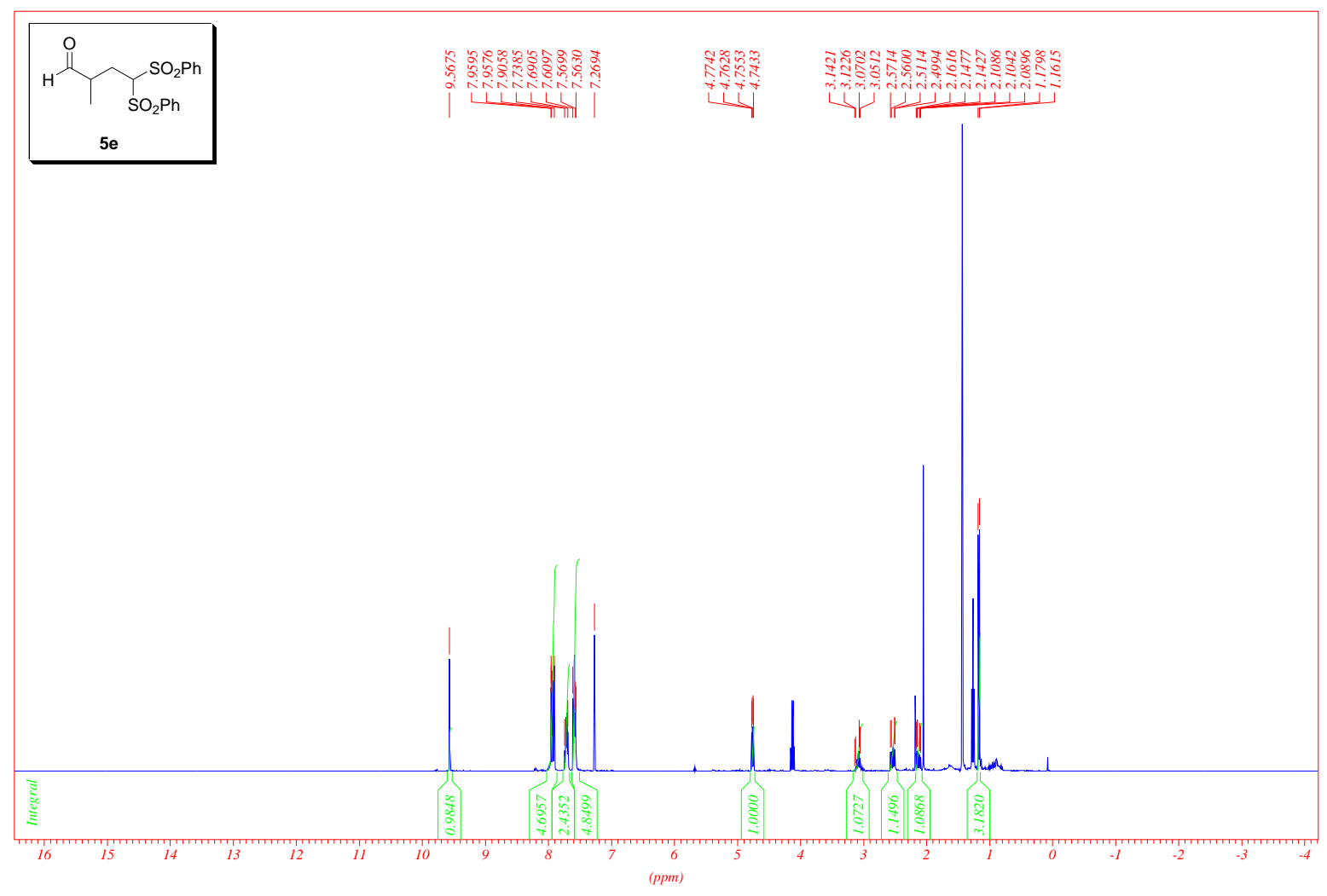

$13 \mathrm{C}(\mathrm{CDCl} 3,100 \mathrm{MHz})$

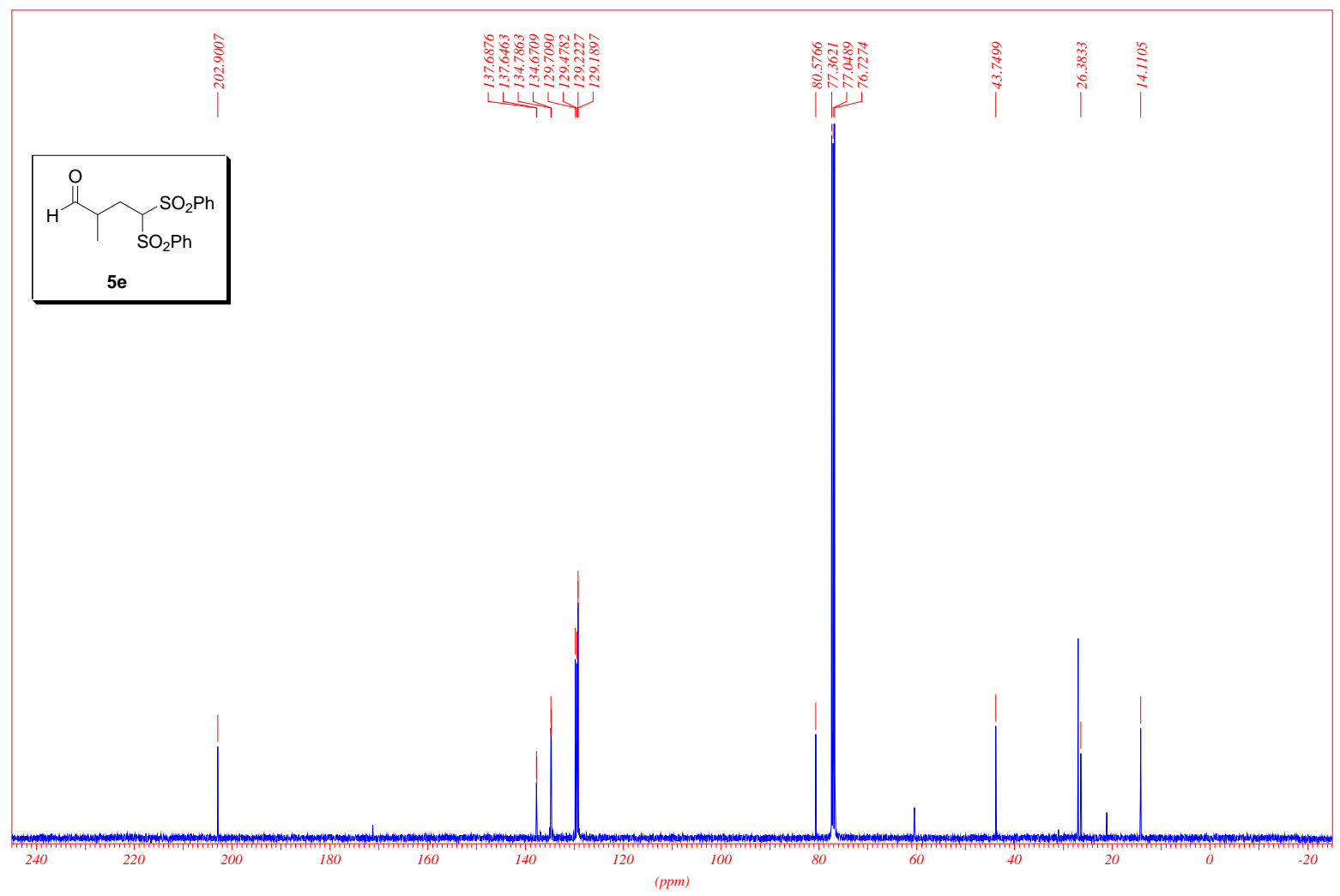


1 H (CDCl3, 400 MHz)

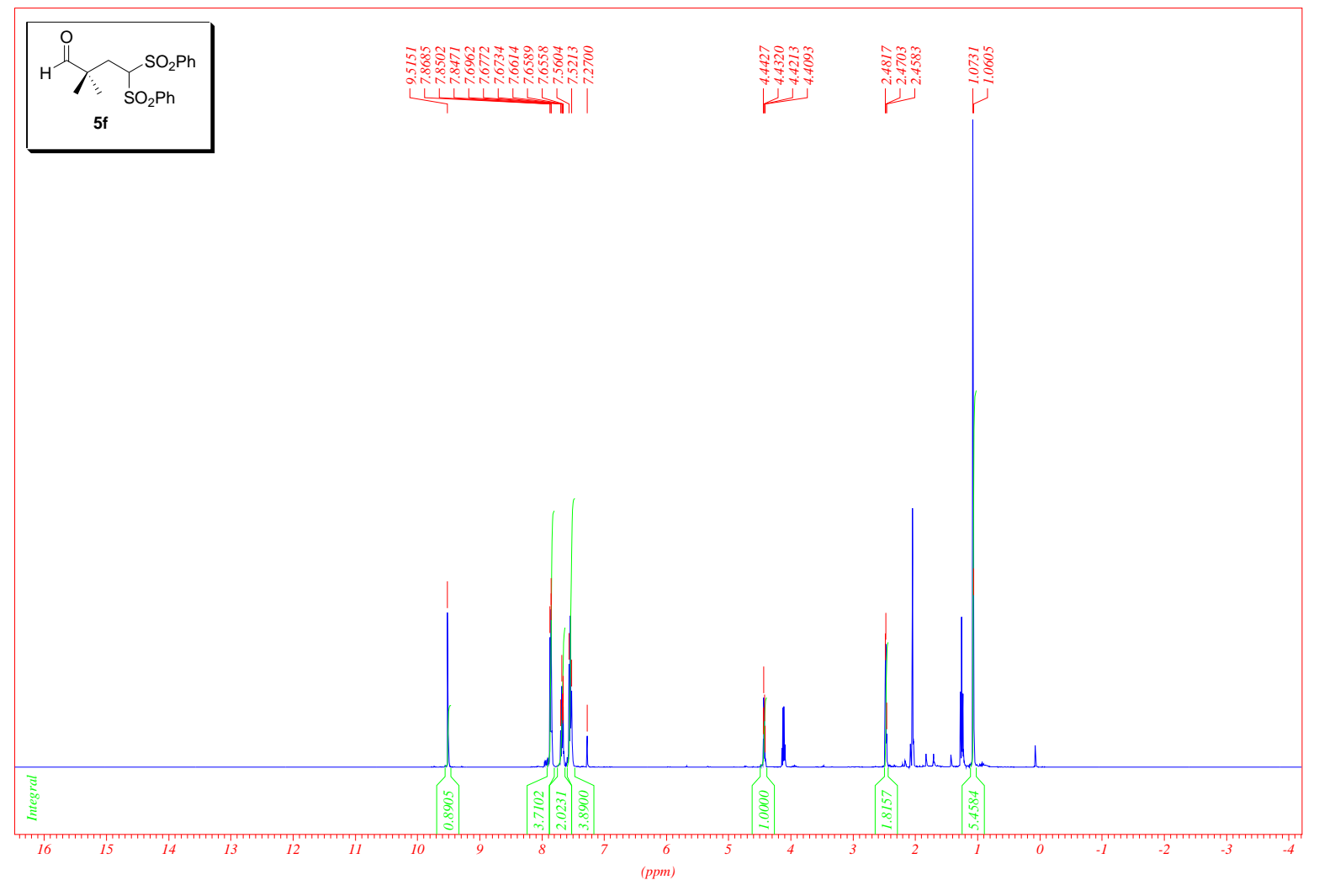

$13 \mathrm{C}(\mathrm{CDCl} 3,100 \mathrm{MHz})$

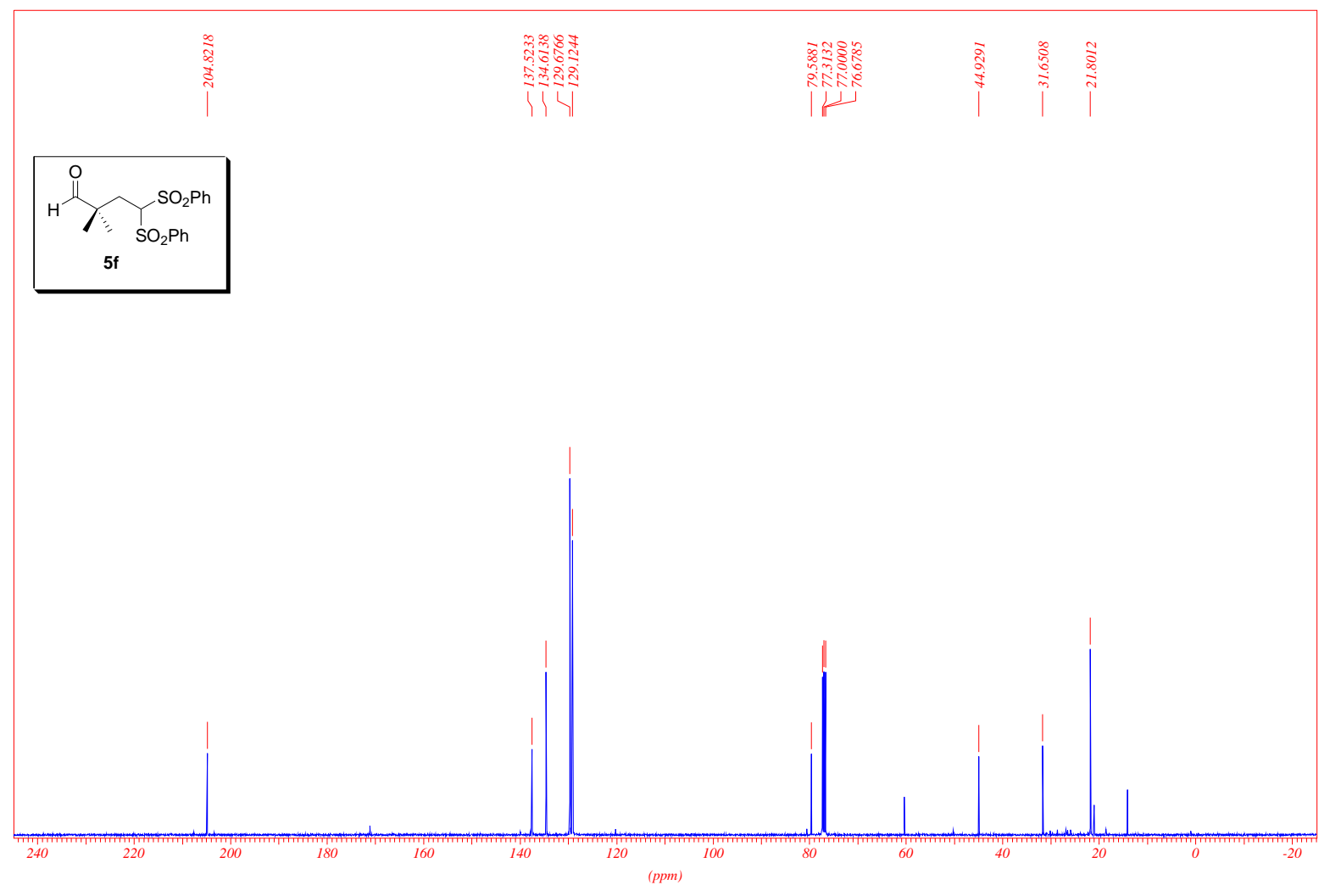




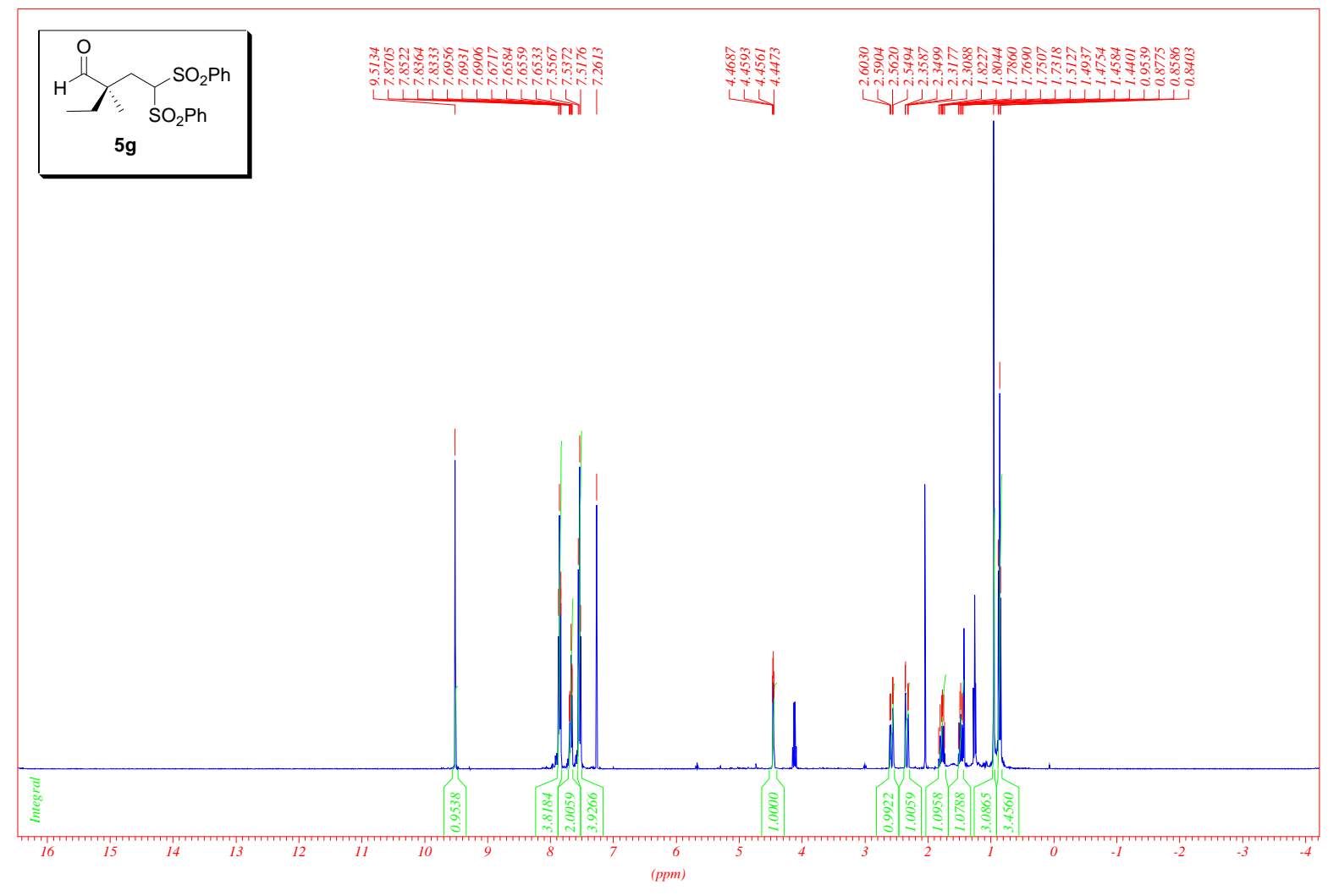

$13 \mathrm{C}$ (CDCl3, $100 \mathrm{MHz})$

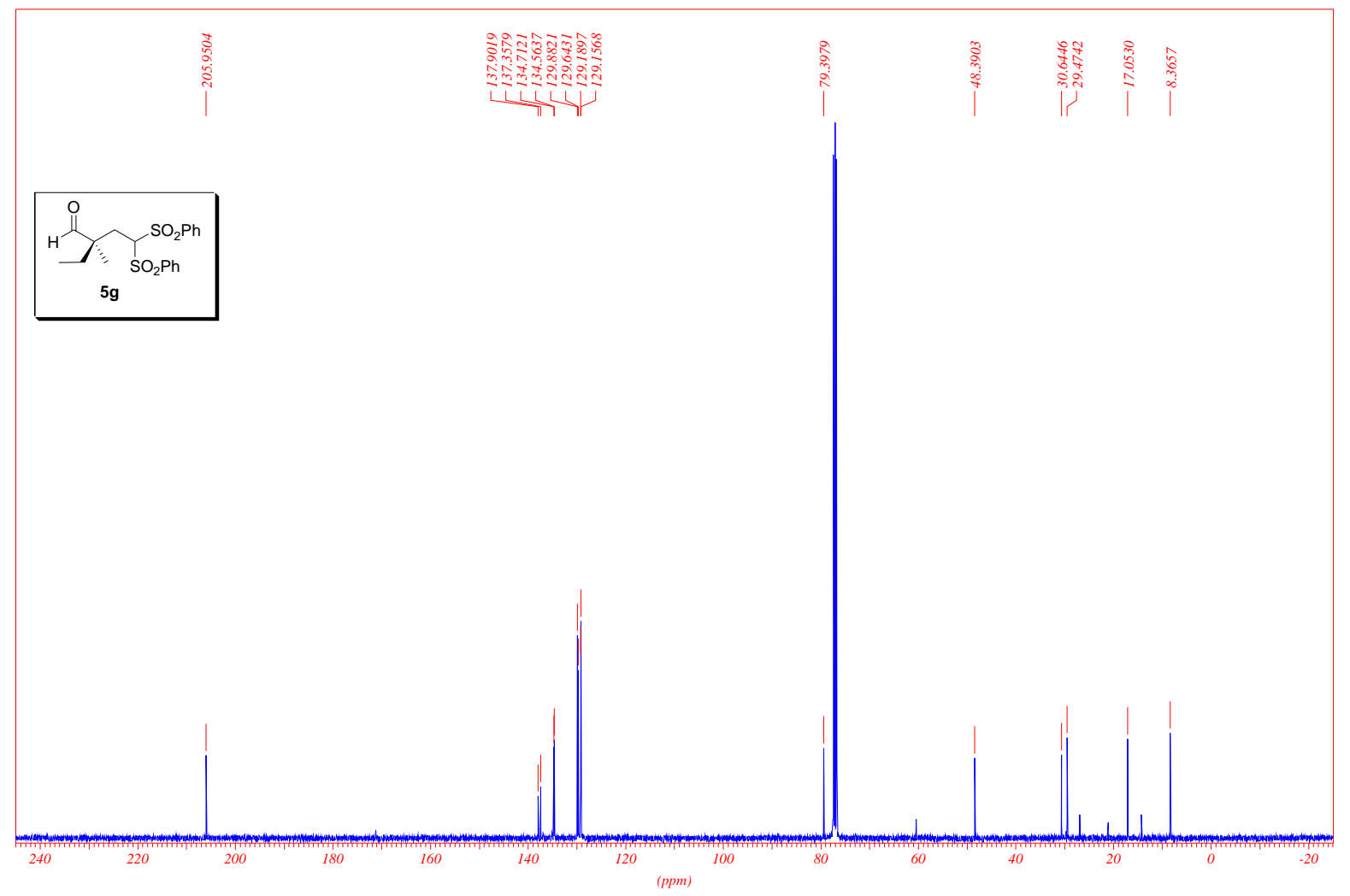



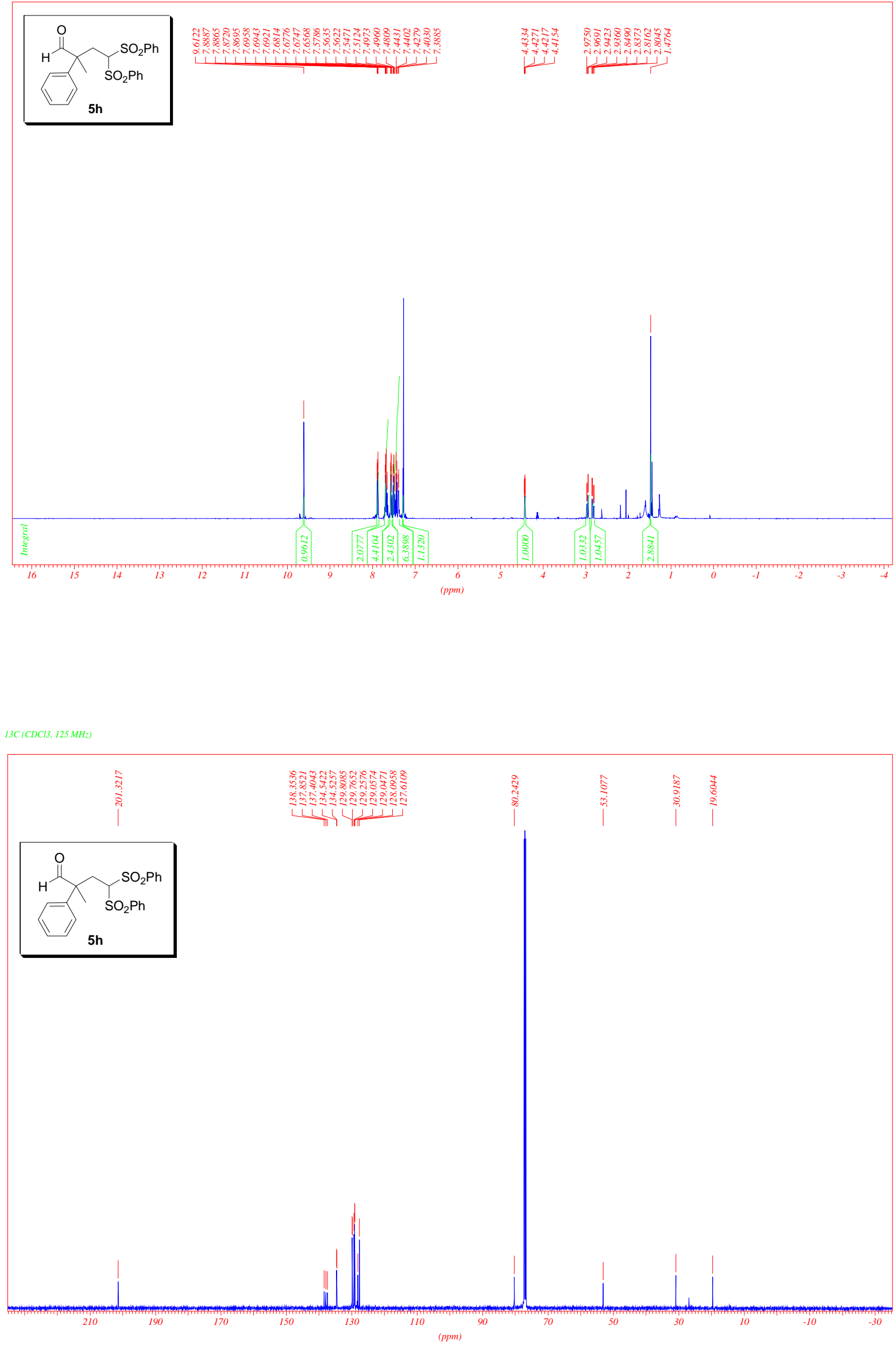


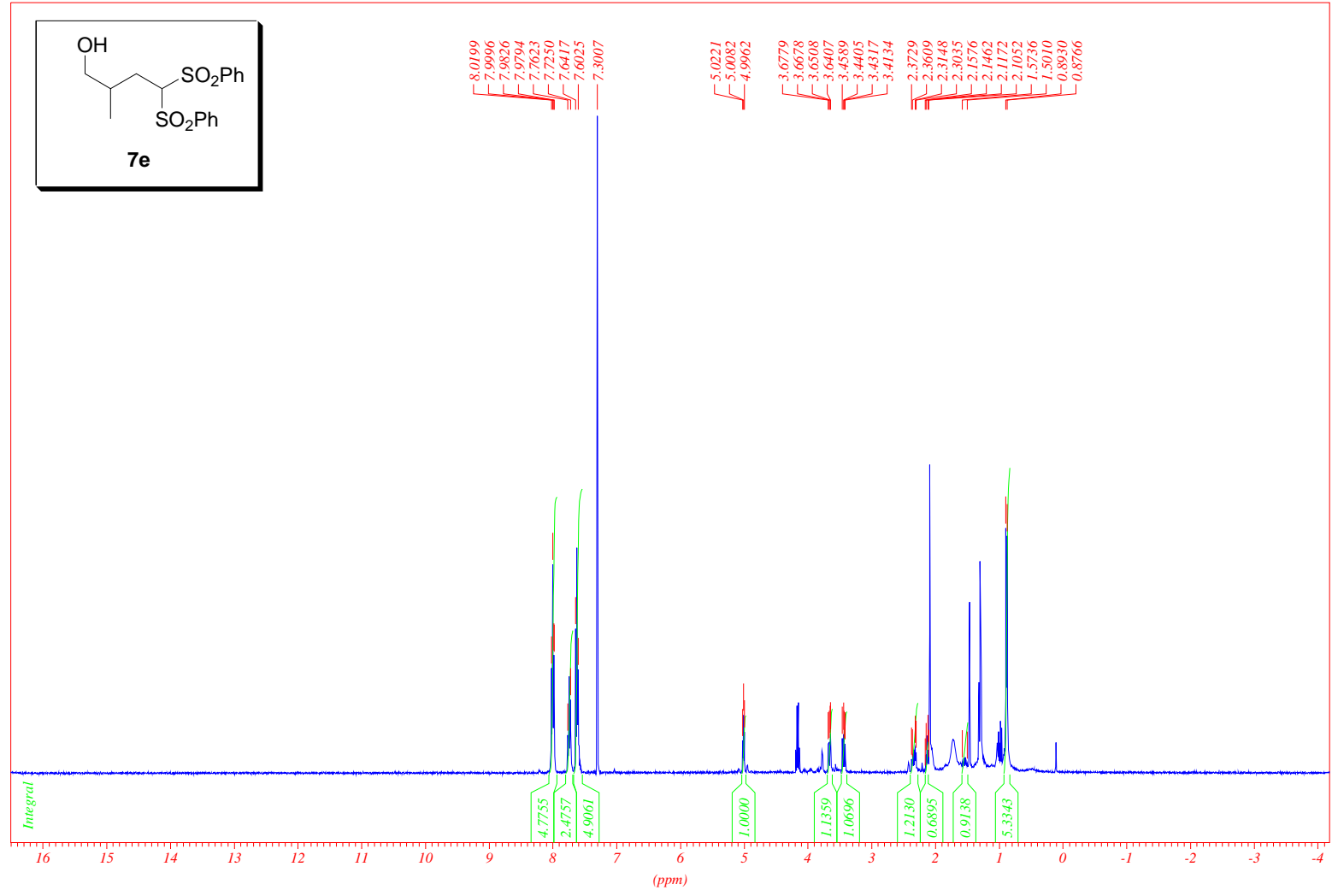

$13 \mathrm{C}$ (CDCl3, $100 \mathrm{MHz})$

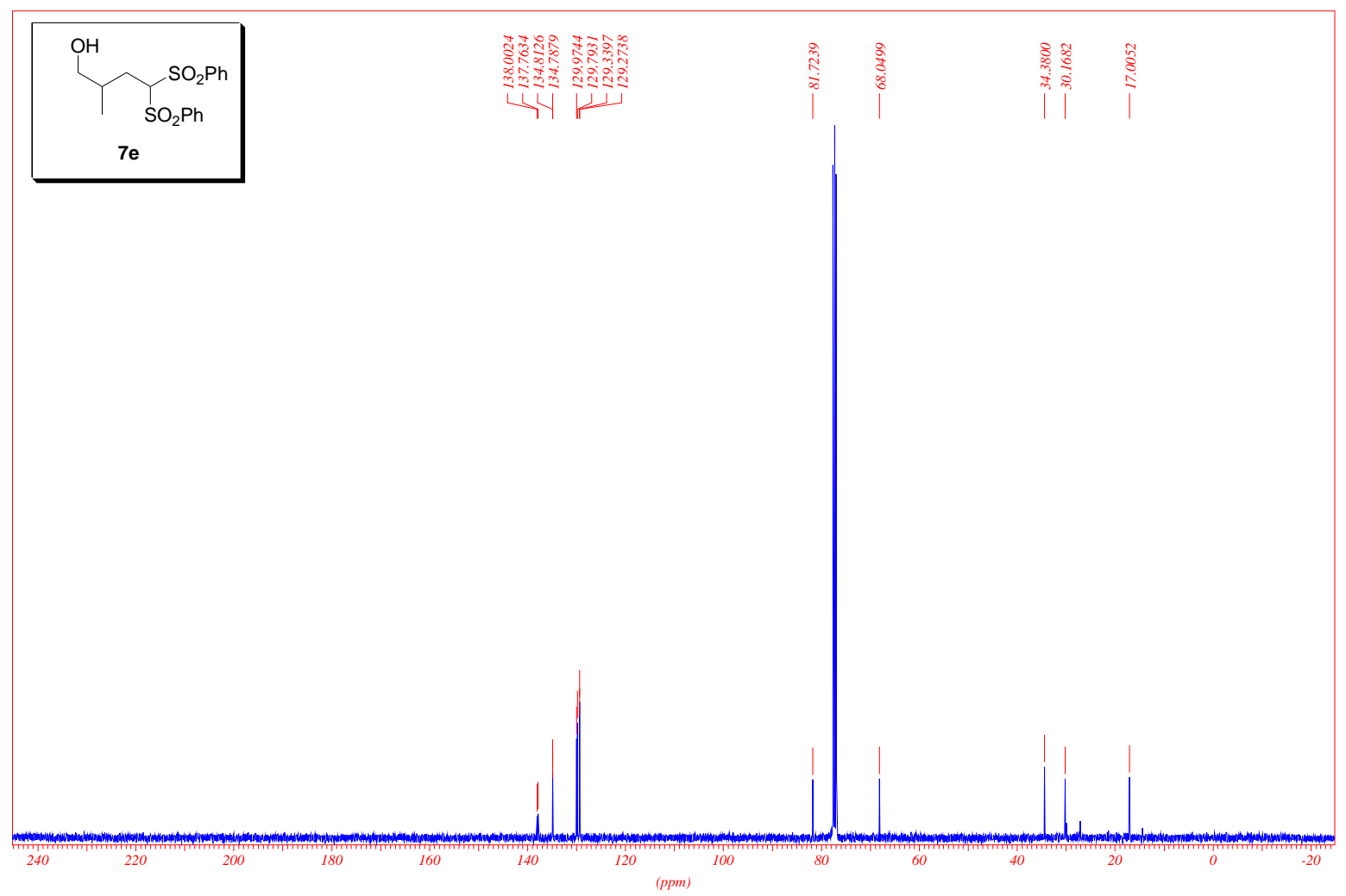



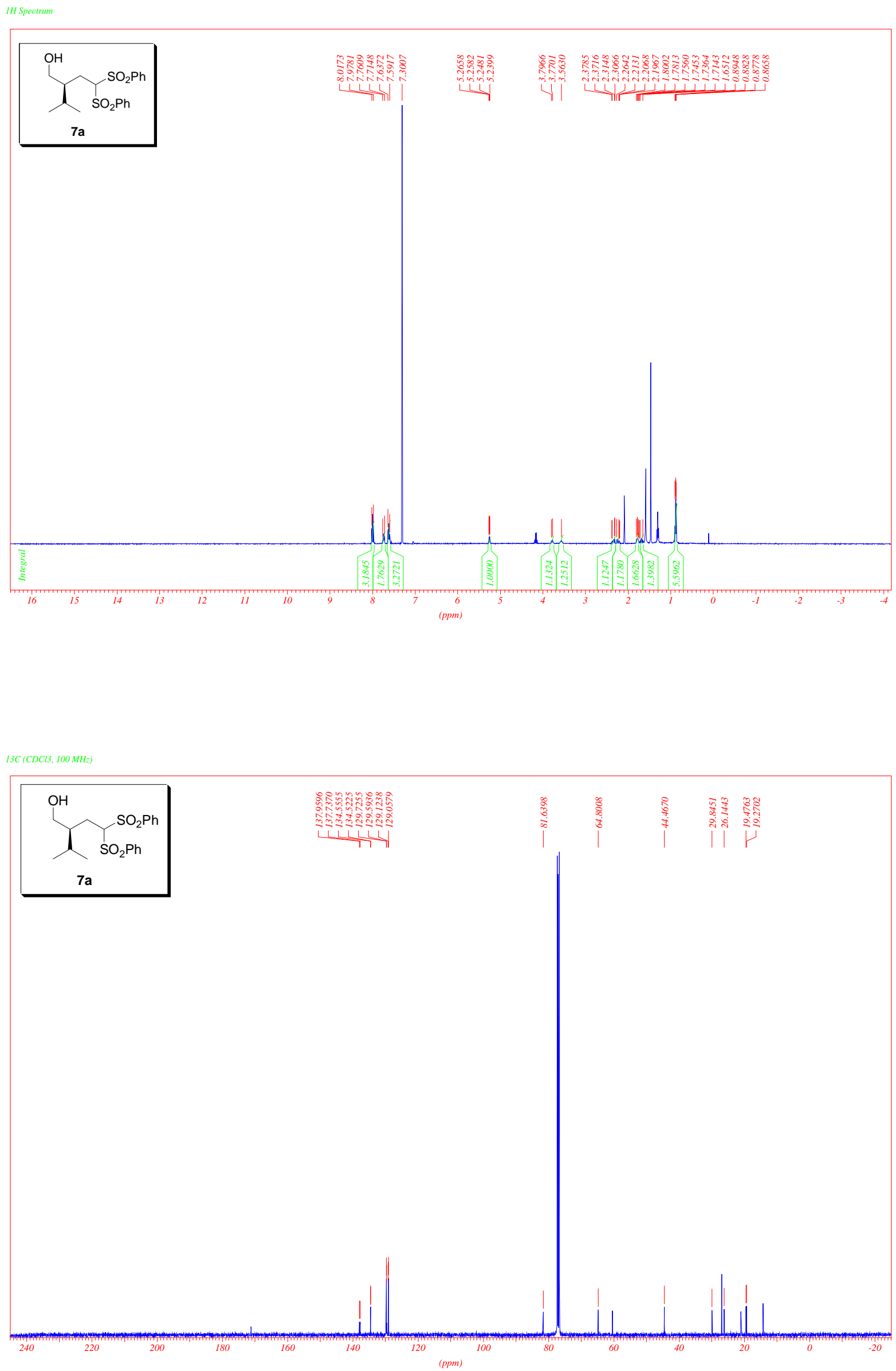
1 (CDCl3, $400 \mathrm{MHz})$

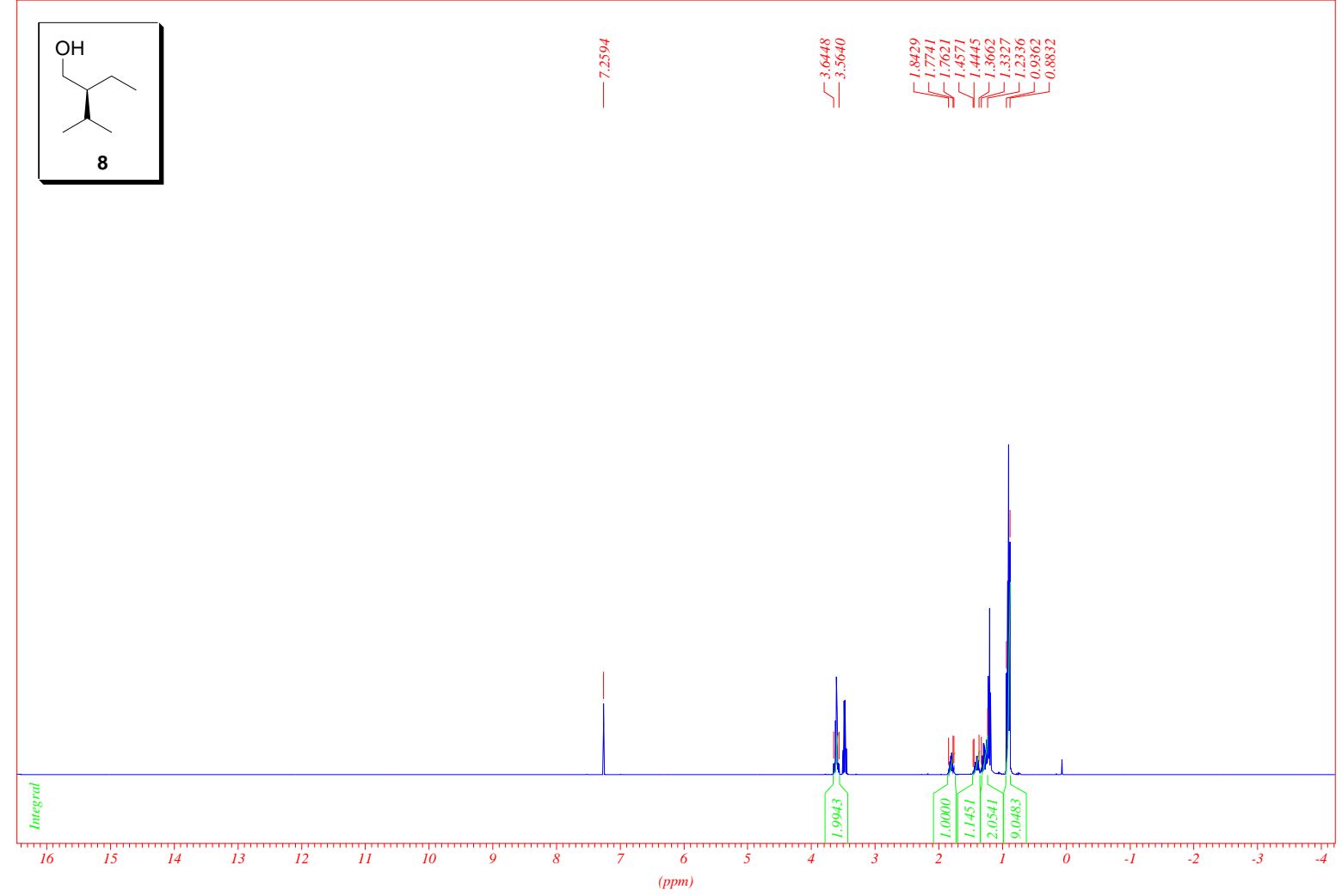

